\title{
Universiteit
}

Leiden

The Netherlands

\section{Subthreshold optical parametric oscillator with nonorthogonal polarization eigenmodes}

Aiello, A.; Nienhuis, G.; Woerdman, J.P.

\section{Citation}

Aiello, A., Nienhuis, G., \& Woerdman, J. P. (2003). Subthreshold optical parametric oscillator with nonorthogonal polarization eigenmodes. Physical Review A, 67, 043803.

doi:10.1103/PhysRevA.67.043803

Version: $\quad$ Not Applicable (or Unknown)

License: $\quad$ Leiden University Non-exclusive license

Downloaded from: https://hdl.handle.net/1887/61250

Note: To cite this publication please use the final published version (if applicable). 


\title{
Subthreshold optical parametric oscillator with nonorthogonal polarization eigenmodes
}

\author{
A. Aiello, G. Nienhuis, and J. P. Woerdman \\ Huygens Laboratory, Leiden University, P.O. Box 9504, Leiden, The Netherlands \\ (Received 3 July 2002; revised manuscript received 27 November 2002; published 8 April 2003)
}

\begin{abstract}
We study the behavior of a type-II degenerate parametric amplifier in a cavity with nonorthogonal polarization eigenmodes. The mode nonorthogonality is achieved by introducing circular birefringence and linear dichroism. We use a scattering matrix formalism to investigate the role of excess quantum noise in such a device. Since only two modes are involved we are able to derive an analytical expression for the twin-photon generation rate measured outside the cavity as a function of the degree of mode nonorthogonality. Contrary to recent claims we conclude that there is no evidence of excess quantum noise for a parametric amplifier working so far below threshold that spontaneous processes dominate. Using the same scattering matrix formalism we also investigate the output spectrum of the amplifier near the threshold of parametric oscillation. We find optical band structures very similar to those known for passive ring cavities. These optical band structures are studied as a function of mode nonorthogonality and mirror reflectivity.
\end{abstract}

DOI: 10.1103/PhysRevA.67.043803

PACS number(s): 42.50.Lc, 42.60.Da, 42.65.Yj

\section{INTRODUCTION}

A linear amplifier is a device that takes an input signal and produces an output signal linearly related to the input signal. Under this definition fall frequency-conserving amplifiers, as laser amplifiers, and frequency-converting amplifiers, as parametric amplifiers. Quantum mechanics sets a lower limit on noise in linear amplifiers [1] which corresponds, in a laser amplifier, to having "one noise photon" in the laser mode (Ref. [2], p. 72) and, in a parametric amplifier, to having "one noise photon" in each of the input modes [3]. This limit is easily reached in small devices, particularly in semiconductor lasers [4]. If the linear amplifier is part of an optical cavity the quantum limit on its performances is strongly affected by the optical characteristics of the cavity itself which offers the possibility to control and to manipulate the quantum noise. This opens a wide range of possible studies which spans from cavity QED (see, e.g., [5] and references therein) to the phenomenon of excess quantum noise [6-13].

Recently there has been a large body of work pointing at the fact that the quantum noise may be enhanced by the so-called excess noise factor or Petermann $K$ factor [6]. From a physical point of view the $K$ factor can be interpreted as if there are $K$ noise photons in the lasing mode instead of the usual "one noise photon." Semiclassically the noise enhancement is due to nonorthogonality of the eigenmodes $[7,14]$. The existence of the Petermann $K$ factor has been experimentally verified in lasers with non-orthogonal eigenmodes, either longitudinal [10], transverse [11], or polarization $[9,12]$ modes, showing that a noise enhancement really occurs. However, the physical origin of this enhancement is under debate; the two main points of view are that it stems from a cavity-enhanced single atom decay rate [15-18] or from an amplification by the gain medium of the spontaneously emitted photons $[13,19,20]$. If the single-atom decay rate were enhanced, excess noise would also be a valid concept (far) below the oscillations threshold of the device under consideration. In this case excess noise could be very useful; for instance, it has been claimed that it could lead to an enhanced generation of twin photons in spontaneous parametric down conversion (SPDC), by placing a nonlinear crystal in an unstable cavity (which has nonorthogonal transverse eigenmodes) [16].

The most common experimental realization of mode nonorthogonality concerns the transverse modes of an unstable cavity. However, this case is intrinsically difficult to treat: one deals with an infinite manifold of transverse modes which cannot be truncated since there is no sharp distinction between system modes (=cavity modes) and reservoir modes ( $=$ free space modes) [21]. This unavoidable difficulty has motivated us to study the effect of excess noise on cavity-enhanced SPDC, for a case where one can construct an exactly solvable quantum theory of mode nonorthogonality. This is possible for a cavity with nonorthogonal polarization eigenmodes (instead of transverse eigenmodes) which has a nonlinear crystal inside.

In fact, SPDC constitutes a natural framework in which to study polarization excess noise in a quantum-mechanical context. Specifically, in a type-II SPDC process, two orthogonally polarized photons are generated. Because of crystal anisotropy, for a fixed frequency only a restricted set of spatial directions is allowed to the emitted photons. In the degenerate case one can achieve a single allowed direction for a collinear emission [22] thus, assuming perfect phase matching, single transverse mode operation can be realized. Although an optical cavity allows, in principle, several resonant longitudinal modes, the double resonance condition (signal and idler) for SPDC restricts this number. It can be shown [23] that, because of crystal birefringence, for a type-II process the double resonance condition can only be satisfied at degenerate frequency so that the number of allowed longitudinal modes is reduced to one.

In this paper we report a detailed study of an optical parametric oscillator with nonorthogonal polarization eigenmodes, extending our previous results of Ref. [24]. Our approach is simple and straightforward: using a scattering matrix formalism we calculate the rate of emitted photons in a SPDC process generated by a type-II degenerate parametric amplifier (DPA) inside a cavity with two nonorthogonal po- 
larization modes, both far below and near the threshold for parametric oscillation [optical parametric oscillator (OPO)]. We use and expand two existing theoretical models, one for the DPA and the other for the cavity, both of which have been experimentally verified. Our conclusion is that there is no enhancement in spontaneous parametric down conversion.

In the second part of this paper we discuss the behavior of the spectrum of a parametric oscillator working close to threshold. We first discuss the definition of spectral resonance within our scattering formalism, then we analyze the OPO spectrum for different cavity realizations. We find a quite unexpected behavior: the OPO spectrum exhibits band structures very similar to those known in passive ring cavities. In fact, we find that because of the mode coupling induced by passive and active optical elements inside the cavity, four resonant peaks per free spectral range appear in the OPO spectrum.

The paper is organized as follows. In Sec. II we introduce a group-theoretical formalism for describing and analyzing the two-mode optical elements which are present in our model in terms of scattering matrices. In Sec. III such formalism is applied to set up the cavity model. We also show explicitly the occurrence of the "geometrical" Petermann $K$ factor in our cavity model. The results obtained in Sec. III are collected and analyzed in Sec. IV where the absence of a $K$-enhanced spontaneous down-conversion rate is proven. In Sec. V we exploit the scattering matrix formalism to investigate the occurrence of band structures in the OPO spectrum in a cavity with nonorthogonal polarization eigenmodes. Finally, we draw conclusions in Sec. VI.

\section{TWO-MODE OPTICAL ELEMENTS AND GROUP THEORY}

The optical devices we consider in this paper are composed of linear and lossless optical elements, and have two input ports (say 1 and 2) and two corresponding output ports. When the elements are passive, no photons are created or destroyed, so that the number of photons entering the two input ports is equal to the number of photons leaving the two output ports. Such devices can be described by a unitary matrix belonging to the group $U(2)$ [25]. Active optical devices can create and annihilate photons but when the difference between the number of photons entering port 1 and that entering port 2 is conserved, the device can be described by a unitary matrix belonging to the group $U(1,1)[26,27]$. In this section we review briefly the matrix representation of lossless passive and active optical devices, characterizing them in terms of $U(2)$ and $U(1,1)$ group properties. We show how, introducing the so-called commutator matrix [28], the Schwinger model for angular momentum can be extended to build the generators of $U(1,1)$ group.

Let us consider a pair of operators $\hat{x}_{1}, \hat{x}_{2}$ which satisfy the following commutation rules:

$$
\left[\hat{x}_{i}, \hat{x}_{j}\right]=0, \quad\left[\hat{x}_{i}, \hat{x}_{j}^{\dagger}\right] \equiv(\Gamma)_{i j} \quad(i, j=1,2),
$$

where $\Gamma$ is a given diagonal $2 \times 2$ matrix. We arrange $\hat{x}_{1}$ and $\hat{x}_{2}$ in a two-dimensional vector $\hat{X}$ (and its adjoint $\hat{X}^{\dagger}$ ) defined as

$$
\hat{X} \equiv\left(\begin{array}{l}
\hat{x}_{1} \\
\hat{x}_{2}
\end{array}\right), \quad \hat{X}^{\dagger} \equiv\left(\begin{array}{ll}
\hat{x}_{1}^{\dagger} & \hat{x}_{2}^{\dagger}
\end{array}\right),
$$

and define the inner product $(-,-)$ between two vectors $\hat{X}$ and $\hat{Y}$ as

$$
(\hat{X}, \hat{Y})=\hat{x}_{i}^{\dagger} \hat{y}_{i} \quad(i=1,2),
$$

where summation over repeated indices is understood. The three Pauli matrices together with the identity matrix form a basis in the vectorial space of $2 \times 2$ matrices; we write them as

$$
\begin{array}{ll}
\sigma_{0}=\left(\begin{array}{ll}
1 & 0 \\
0 & 1
\end{array}\right), & \sigma_{1}=\left(\begin{array}{ll}
0 & 1 \\
1 & 0
\end{array}\right), \\
\sigma_{2}=\left(\begin{array}{cc}
0 & -i \\
i & 0
\end{array}\right), & \sigma_{3}=\left(\begin{array}{cc}
1 & 0 \\
0 & -1
\end{array}\right) .
\end{array}
$$

Using the Pauli matrices we can construct four Hermitian operators defined as

$$
\hat{S}_{a} \equiv\left(\hat{X}, \sigma_{a} \hat{X}\right) \quad(a=0, \ldots, 3) .
$$

These operators satisfy the following commutation rules:

$$
\left[\hat{S}_{a}, \hat{S}_{b}\right]=\left(\hat{X}, \sigma_{a b} \hat{X}\right) \quad(a, b=0, \ldots, 3),
$$

where

$$
\sigma_{a b} \equiv \sigma_{a} \Gamma \sigma_{b}-\sigma_{b} \Gamma \sigma_{a} \quad(a, b=0, \ldots, 3) .
$$

Because of completeness of the set of Pauli matrices, Eq. (4), we can always write, choosing adequately the constants $f_{a b c}$,

$$
\sigma_{a b}=i f_{a b c} \sigma_{c} \quad(a, b, c=0, \ldots, 3) .
$$

Using Eqs. (8) we can then write Eq. (6) as

$$
\left[\hat{S}_{a}, \hat{S}_{b}\right]=i f_{a b c} \hat{S}_{c},
$$

which shows that the four operators $\hat{S}_{a}$ satisfy the same commutation relations as the generators of a symmetry group. The numbers $f_{a b c}$ are called structure constants and completely determine the group multiplication law [29]. The operators $\hat{S}_{a}$ generate transformation of the vector operator $\hat{X}$, in the form

$$
\exp \left(z \hat{S}_{a}\right) \hat{X} \exp \left(-z \hat{S}_{a}\right)=\exp \left(-z \Gamma \sigma_{a}\right) \hat{X},
$$

which follows after differentiation with respect to $z$, while using the identity $\left[\hat{S}_{a}, \hat{X}\right]=-\Gamma \sigma_{a} \hat{X}$. From Eqs. (6) and (7) 
one notices that $\left[\hat{S}_{0}, \hat{S}_{a}\right]=0$ when $\Gamma=\sigma_{0}$, and that $\left[\hat{S}_{3}, \hat{S}_{a}\right]$ $=0$ when $\Gamma=\sigma_{3}$. These two cases are realized when one chooses

$$
\hat{X}=\left(\begin{array}{c}
\hat{a} \\
\hat{b}
\end{array}\right) \quad \text { or } \quad \hat{X}=\left(\begin{array}{c}
\hat{a} \\
\hat{b}^{\dagger}
\end{array}\right)
$$

where $\hat{a}, \hat{b}$ are independent harmonic oscillator operators which satisfy the boson commutation relations:

$$
\begin{aligned}
& {[\hat{a}, \hat{b}]=0=\left[\hat{a}, \hat{b}^{\dagger}\right],} \\
& {\left[\hat{a}, \hat{a}^{\dagger}\right]=1=\left[\hat{b}, \hat{b}^{\dagger}\right] .}
\end{aligned}
$$

Case 1: $\Gamma=\sigma_{0}$. In this case the operators $\hat{S}_{a}$ belong to the Lie algebra of the group $U(2)$ and we recover the Schwinger representation of two modes,

$$
\begin{gathered}
\hat{N}_{a}+\hat{N}_{b}=\hat{S}_{0}=\hat{a}^{\dagger} \hat{a}+\hat{b}^{\dagger} \hat{b}, \\
\hat{J}_{x}=\frac{\hat{S}_{1}}{2}=\frac{1}{2}\left(\hat{a}^{\dagger} \hat{b}+\hat{b}^{\dagger} \hat{a}\right), \\
\hat{J}_{y}=\frac{\hat{S}_{2}}{2}=-\frac{i}{2}\left(\hat{a}^{\dagger} \hat{b}-\hat{b}^{\dagger} \hat{a}\right), \\
\hat{J}_{z}=\frac{\hat{S}_{3}}{2}=\frac{1}{2}\left(\hat{a}^{\dagger} \hat{a}-\hat{b}^{\dagger} \hat{b}\right) .
\end{gathered}
$$

The operators $\hat{J}_{x}, \hat{J}_{y}, \hat{J}_{z}$ obey the usual commutation rules of angular momentum $\left[\hat{J}_{x}, \hat{J}_{y}\right]=i \hat{J}_{z}$, etc. The conserved quantity associated with $\hat{S}_{0}$ is the total number of photons represented by the operator $\hat{N}_{a}+\hat{N}_{b}$ which commutes with the three angular momentum operators $\hat{J}_{x}, \hat{J}_{y}, \hat{J}_{z}$.

In order to see explicitly the connection between lossless passive optical devices and the elements of the group $U(2)$ we denote with $\hat{x}_{1}$ and $\hat{x}_{2}$ the annihilation operators for the field entering the two input ports and with $\hat{y}_{1}$ and $\hat{y}_{2}$ the annihilation operators for the field leaving the two output ports. These four operators are connected by a scattering matrix $\mathbf{M}$ whose form is

$$
\left(\begin{array}{l}
\hat{y}_{1} \\
\hat{y}_{2}
\end{array}\right)=\left(\begin{array}{ll}
M_{11} & M_{12} \\
M_{21} & M_{22}
\end{array}\right)\left(\begin{array}{c}
\hat{x}_{1} \\
\hat{x}_{2}
\end{array}\right) .
$$

Conservation of probability in a scattering process demands that output operators satisfy the same commutation relations as the input operators. This requirement leads to the unitarity condition for $\mathbf{M}$,

$$
\mathbf{M M}^{\dagger}=\mathbf{1}
$$

Here we write explicitly some scattering matrices and the associate transformations that will be used in the next section. The operators $\hat{J}_{x}$ and $\hat{J}_{y}$ generate two possible scattering matrices for a beam splitter and/or a rotator [30],

$$
\begin{gathered}
e^{i \alpha \hat{J}_{x}}\left(\begin{array}{l}
\hat{a} \\
\hat{b}
\end{array}\right) e^{-i \alpha \hat{J}_{x}}=\left(\begin{array}{cc}
\cos (\alpha / 2) & -i \sin (\alpha / 2) \\
-i \sin (\alpha / 2) & \cos (\alpha / 2)
\end{array}\right)\left(\begin{array}{l}
\hat{a} \\
\hat{b}
\end{array}\right), \\
e^{i \beta \hat{J}_{y}}\left(\begin{array}{c}
\hat{a} \\
\hat{b}
\end{array}\right) e^{-i \beta \hat{J}_{y}}=\left(\begin{array}{cc}
\cos (\beta / 2) & -\sin (\beta / 2) \\
\sin (\beta / 2) & \cos (\beta / 2)
\end{array}\right)\left(\begin{array}{l}
\hat{a} \\
\hat{b}
\end{array}\right),
\end{gathered}
$$

while the scattering matrix accounting for free-field propagation is generated by operator $\hat{J}_{z}$,

$$
e^{i \gamma \hat{J}_{z}}\left(\begin{array}{l}
\hat{a} \\
\hat{b}
\end{array}\right) e^{-i \gamma \hat{J}_{z}}=\left(\begin{array}{cc}
e^{-i \gamma / 2} & 0 \\
0 & e^{i \gamma / 2}
\end{array}\right)\left(\begin{array}{l}
\hat{a} \\
\hat{b}
\end{array}\right) .
$$

Case 2: $\Gamma=\sigma_{3}$. Using Eq. (5) it is easy to see that the operators $\hat{S}_{a}$ belong to the Lie algebra of the group $U(1,1)$,

$$
\begin{gathered}
\hat{N}_{a}-\hat{N}_{b}-1=\hat{S}_{3}=\hat{a}^{\dagger} \hat{a}-\hat{b} \hat{b}^{\dagger}, \\
\hat{K}_{x}=\frac{\hat{S}_{1}}{2}=\frac{1}{2}\left(\hat{a}^{\dagger} \hat{b}^{\dagger}+\hat{b} \hat{a}\right), \\
\hat{K}_{y}=\frac{\hat{S}_{2}}{2}=-\frac{i}{2}\left(\hat{a}^{\dagger} \hat{b}^{\dagger}-\hat{b} \hat{a}\right), \\
\hat{K}_{z}=\frac{\hat{S}_{0}}{2}=\frac{1}{2}\left(\hat{a}^{\dagger} \hat{a}+\hat{b} \hat{b}^{\dagger}\right) .
\end{gathered}
$$

In this case the difference in photon number is conserved, that is the operator $\hat{N}_{a}-\hat{N}_{b}$ commutes with $\hat{K}_{x}, \hat{K}_{y}, \hat{K}_{z}$ which are generators of the group $S U(1,1)$. The commutation rules for these operators are $\left[\hat{K}_{x}, \hat{K}_{y}\right]=-i \hat{K}_{z},\left[\hat{K}_{y}, \hat{K}_{z}\right]$ $=i \hat{K}_{x}, \quad\left[\hat{K}_{z}, \hat{K}_{x}\right]=i \hat{K}_{y}$. The scattering matrices generated by the $S U(1,1)$ operators follow by Eq. (10). They take the explicit form

$$
\begin{gathered}
e^{i \alpha \hat{K}_{x}}\left(\begin{array}{c}
\hat{a} \\
\hat{b}^{\dagger}
\end{array}\right) e^{-i \alpha \hat{K}_{x}}=\left(\begin{array}{cc}
\cosh (\alpha / 2) & -i \sinh (\alpha / 2) \\
i \sinh (\alpha / 2) & \cosh (\alpha / 2)
\end{array}\right)\left(\begin{array}{c}
\hat{a} \\
\hat{b}^{\dagger}
\end{array}\right), \\
e^{i \beta \hat{K}_{y}}\left(\begin{array}{c}
\hat{a} \\
\hat{b}^{\dagger}
\end{array}\right) e^{-i \beta \hat{K}_{y}}=\left(\begin{array}{cc}
\cosh (\beta / 2) & \sinh (\beta / 2) \\
\sinh (\beta / 2) & \cosh (\beta / 2)
\end{array}\right)\left(\begin{array}{c}
\hat{a} \\
\hat{b}^{\dagger}
\end{array}\right) \\
e^{i \gamma \hat{K}_{z}}\left(\begin{array}{c}
\hat{a} \\
\hat{b}^{\dagger}
\end{array}\right) e^{-i \gamma \hat{K}_{z}}=\left(\begin{array}{cc}
e^{i \gamma / 2} & 0 \\
0 & e^{-i \gamma / 2}
\end{array}\right)\left(\begin{array}{c}
\hat{a} \\
\hat{b}^{\dagger}
\end{array}\right)
\end{gathered}
$$

Using the last two equations we can construct the scattering matrix representing the nonlinear crystal, as shown in Ref. [26].

\section{THE CAVITY MODEL}

We now apply the formalism developed in Sec. II to describe a cavity with nonorthogonal polarization modes. Our 


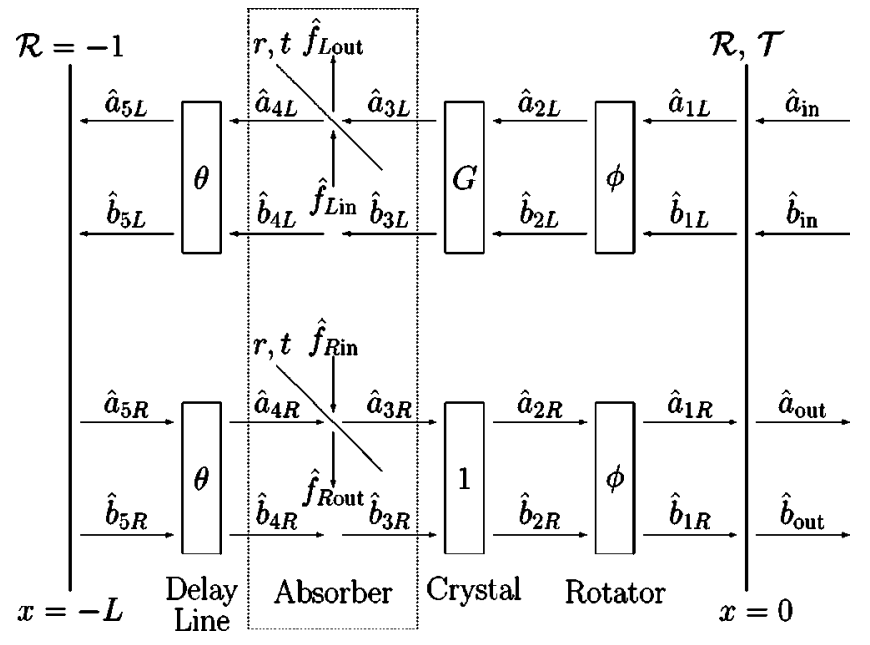

FIG. 1. Schematic representation of the degenerate-cavity parametric amplifier. Modes $a$ and $b$ have orthogonal polarizations. The boxes indicated with $\phi, G$, and $\theta$ represent the rotator, the nonlinear crystal, and the delay line, respectively. In the dotted box we show the absorber modeled as a beam splitter acting only on mode $a$. For right-traveling modes we have put $G=1$ to indicate the passive crystal behavior when there is no phase matching.

model of a degenerate parametric amplifier inside a FabryPérot cavity is, in fact, an extension of the model of Gardiner et al. [31] to the case of a cavity with nonorthogonal polarization modes.

\section{A. Scattering matrix for a cavity round-trip}

We consider a cavity having one perfectly reflecting mirror at position $x=-L$, and a partially reflecting mirror at $x$ $=0$, as shown in Fig. 1 .

We decompose the electric field inside the cavity into left (subscript $L$ ) and right (subscript $R$ ) propagating waves. In degenerate type-II down-conversion two orthogonally polarized modes are excited at the same frequency $\omega=\Omega / 2$, where $\Omega$ is the frequency of the pump field. Let us denote with $a$ and $b$ these two field modes and assume that their polarization is parallel to the $y$ and $z$ axis, respectively. Another mode $f$ (also decomposed in $f_{L}$ and $f_{R}$ parts), is introduced in order to assure the unitarity of the model; we call this mode the noise mode. We assume that mode $f$ has the same polarization as mode $a$. The role of this noise mode will be soon made clear; for the moment we describe, as in Ref. [8], the DPA cavity using a scattering matrix which is unitary only when it accounts both for field and noise modes. We shall see that nonorthogonality of the cavity modes naturally appears as a consequence of restricting the scattering matrix to the set of field modes $a$ and $b$. However, truncating the scattering matrix to the field modes is not enough to achieve mode nonorthogonality; it is necessary to introduce a non-Hermitian coupling between them. In our model the mode nonorthogonality is achieved by inserting in the cavity a phase anisotropy due to circular birefringence (polarization rotator) and a loss anisotropy generated by linear dichroism (polarization-dependent absorber), following the scheme given in [9]. Another way to produce nonorthogonal polar- ization modes is to use linear birefringence and linear dichroism at $45^{\circ}$ as in Ref. [12]. Although these two alternative ways are implemented using physically different devices, both lead to basically the same expressions for the Petermann $K$ factor, as we shall see in Sec. III B.

The canonical quantization scheme requires us to express the electromagnetic field inside the cavity in terms of a continuous or discrete complete set of functions $\left\{u_{n}\right\}$ (the eigenmodes of the cavity) and associating with them a corresponding set of field operators $\left\{\hat{a}_{n}\right\}$. A serious problem arises when the set of cavity eigenmodes $\left\{u_{n}\right\}$ is not orthogonal. In fact, as shown in Refs. [8,13], a set of nonorthogonal modes cannot be turned into a set of noncommuting operators. In order to avoid this problem our calculations are based on the orthogonal sets of operators $\left\{\hat{\alpha}_{\text {in }}, \hat{f}_{\alpha_{\text {in }}}\right\},\left\{\hat{\alpha}_{\text {out }}, \hat{f}_{\alpha_{\text {ou }}}\right\}$ ( $\alpha$ $=a, b)$ associated with a corresponding set of plane-wave modes [31]. We assume that the input and output operators satisfy the usual (discrete) commutation relations

$$
\begin{gathered}
{\left[\hat{a}_{\mathrm{x}}, \hat{b}_{\mathrm{x}}\right]=0=\left[\hat{a}_{\mathrm{x}}, \hat{b}_{\mathrm{x}}^{\dagger}\right],} \\
{\left[\hat{a}_{\mathrm{x}}, \hat{a}_{\mathrm{x}}^{\dagger}\right]=1=\left[\hat{b}_{\mathrm{x}}, \hat{b}_{\mathrm{x}}^{\dagger}\right]} \\
(\mathrm{x}=\text { in, out }),
\end{gathered}
$$

and similarly for the noise operators.

The optical elements inside the cavity are as follows: an absorber modeled as a beam splitter acting only on mode $a(y$ polarization), a crystal with nonlinear gain $G$, and a rotator which rotates the polarization axes by an angle $\phi$ along the $x$ axis. The propagation of the modes over a cavity with length $L$ is modeled by a delay line in front of the left mirror which introduces a phase shift $\theta=\omega L / c$. We assume that all optical elements are infinitesimally thin and that the operator phases at the position $x=0$ are equal to zero. The scattering matrices for the various optical elements inside the cavity are given below. On the output mirror the input annihilation operators belonging to the $a$ mode are related to the input operators on the same mode, by the transformation

$$
\begin{aligned}
& \hat{a}_{\text {out }}=\mathcal{T} \hat{a}_{1 R}+\mathcal{R} \hat{a}_{\text {in }}, \\
& \hat{a}_{1 L}=\mathcal{R} \hat{a}_{1 R}+\mathcal{T} \hat{a}_{\text {in }},
\end{aligned}
$$

where $\mathcal{R}=-\sqrt{R}, \mathcal{T}=i \sqrt{1-R}$, and $0 \leqslant R<1$. For the mode $b$ the above relations hold if we make everywhere the substitution $a \rightarrow b$. The effect of the rotator on left-traveling mode operators can be represented as [30]

$$
\begin{aligned}
& \hat{a}_{2 L}=\cos \phi \hat{a}_{1 L}+\sin \phi \hat{b}_{1 L}, \\
& \hat{b}_{2 L}=-\sin \phi \hat{a}_{1 L}+\cos \phi \hat{b}_{1 L} .
\end{aligned}
$$

The corresponding matrix for right-traveling modes is obtained substituting in the above formula $1 \leftrightarrow 2$ and $L \rightarrow R$. Note that we have chosen as a rotator, a device antisymmetric with respect to temporal inversion [32] (e.g., a Faraday rotator); then the total rotation angle is doubled after a round trip. For completeness we note that in case of a device which 
is symmetric with respect to temporal inversion (e.g., a quartz crystal which displays optical activity), the light beam inside the cavity would retrieve its original polarization after one round trip. Polarization rotation can be also achieved using a half wave plate which introduces a $\pi$ phase difference between fast and slow ( $f$ and $s$ ) axes [33]. This device has been used jointly with a linear dichroic element with its axes at $\pm 45^{\circ}$ with respect to $f$ and $s$ in Refs. [12,34]. However, we have preferred to use a Faraday rotator, jointly with a linear dichroic element with its axes parallel to the $a$ and $b$ polarization directions, since this configuration leads to a more clear separation between the phase anisotropy and the loss anisotropy inside the cavity.

The scattering matrix for the parametric crystal, in the nondepleted pump approximation [31,35], is given by

$$
\begin{aligned}
& \hat{a}_{3 L}=G \hat{a}_{2 L}+\left(G^{2}-1\right)^{1 / 2} \hat{b}_{2 L}^{\dagger}, \\
& \hat{b}_{3 L}^{\dagger}=\left(G^{2}-1\right)^{1 / 2} \hat{a}_{2 L}+G \hat{b}_{2 L}^{\dagger},
\end{aligned}
$$

where the real-valued gain $G$ satisfies $G>1$. For the righttraveling modes the crystal is transparent due to the absence of phase matching and in this case the operator transformations can be obtained from Eqs. (26) after the substitutions $3 \leftrightarrow 2, L \rightarrow R$, and $G=1$. Since Eqs. (26) preserve bosonic commutation rules it is not necessary, for a parametric amplifier with a classical nondepleted pump, to add noise from an external bath [1] to account for pump fluctuations. In our model only the down-converted field is confined by the cavity, not the pump field, therefore the cavity mode structure cannot affect the pump beam fluctuations. Incidentally, we note that when using this scattering matrix formalism, the difference between a linear and a nonlinear amplifier is rooted only in the choice of the operators which are coupled by the matrix, but not in the matrix itself, which is the same in both cases. In fact, in a linear amplifier the nondiagonal matrix elements couple a field annihilation operator with a noise creation operator, while in a nonlinear amplifier the coupling is between two different field modes, as in Eqs. (26).

The scattering matrix representing the absorber, which introduces losses only for the mode $a$, is written as [36]

$$
\begin{gathered}
\hat{a}_{4 L}=t \hat{a}_{3 L}+r \hat{f}_{L \text { in }}, \\
\hat{b}_{4 L}=\hat{b}_{3 L}, \\
\hat{f}_{L \text { out }}=r \hat{a}_{3 L}+t \hat{f}_{L \text { in }},
\end{gathered}
$$

where $r=i \sqrt{1-t^{2}}$ and the real parameter $t(0 \leqslant t \leqslant 1)$ represents the ratio between field amplitudes along $y$ and $z$ polarization directions. For right-traveling modes we obtain essentially the same equations by substituting $4 \leftrightarrow 3$ and $L$ $\rightarrow R$, that is we consider a device insensitive with respect to the direction of the impinging light. We note that truncating the transformation equations (27) to the field modes only leads to the following nonunitary transformation:

$$
\left(\begin{array}{l}
\hat{a}_{4 \mathrm{~L}} \\
\hat{b}_{4 \mathrm{~L}}
\end{array}\right)=\left(\begin{array}{ll}
t & 0 \\
0 & 1
\end{array}\right)\left(\begin{array}{l}
\hat{a}_{3 \mathrm{~L}} \\
\hat{b}_{3 \mathrm{~L}}
\end{array}\right) .
$$

Since as the absorber we have chosen a linear dichroic element with its axes parallel to the $a$ and $b$ polarization directions, it introduces only anisotropic losses but no phase anisotropy and therefore the matrix Eq. (28) is diagonal.

The delay line with phase shift $\theta$ can be simply represented as

$$
\begin{aligned}
& \hat{a}_{5 L}=\exp (i \theta) \hat{a}_{4 L}, \\
& \hat{a}_{4 R}=\exp (i \theta) \hat{a}_{5 R},
\end{aligned}
$$

where $\theta=\omega L / c$. It allows us to evaluate the effects of the cavity length $L$. The same relations hold for mode $b$. Finally, on the left mirror the boundary condition requires

$$
\hat{a}_{5 R}=-\hat{a}_{5 L},
$$

and similarly for mode $b$.

Equations (25)-(31) can be straightforwardly solved to express right-traveling mode operators in terms of lefttraveling mode operators,

$$
\begin{aligned}
\left(\begin{array}{c}
\hat{a}_{1 R} \\
\hat{b}_{1 R}
\end{array}\right)= & G\left(\begin{array}{cc}
-\gamma_{+} \cos (2 \phi)-\gamma_{-} & -\gamma_{+} \sin (2 \phi) \\
\gamma_{+} \sin (2 \phi) & -\gamma_{+} \cos (2 \phi)+\gamma_{-}
\end{array}\right) \\
& \times\left(\begin{array}{c}
\hat{a}_{1 L} \\
\hat{b}_{1 L}
\end{array}\right)+\left(G^{2}-1\right)^{1 / 2} \\
& \times\left(\begin{array}{cc}
\gamma_{-} \sin (2 \phi) & -\gamma_{-} \cos (2 \phi)-\gamma_{+} \\
\gamma_{-} \cos (2 \phi)-\gamma_{+} & \gamma_{-} \sin (2 \phi)
\end{array}\right) \\
& \times\left(\begin{array}{c}
\hat{a}_{1 L}^{\dagger} \\
\hat{b}_{1 L}^{\dagger}
\end{array}\right)+\left(\begin{array}{c}
\hat{f}_{a} \\
\hat{f}_{b}
\end{array}\right),
\end{aligned}
$$

where $\gamma_{ \pm}=\exp (2 i \theta)\left(t^{2} \pm 1\right) / 2$ and

$$
\begin{aligned}
& \hat{f}_{a}=r\left(t \hat{f}_{L \text { in }}+\hat{f}_{R \text { in }}\right) \cos \phi, \\
& \hat{f}_{b}=-r\left(t \hat{f}_{L \text { in }}+\hat{f}_{R \text { in }}\right) \sin \phi .
\end{aligned}
$$

The effect of the noise on mode $b$ appears as a consequence of introducing the rotator: $\hat{f}_{b}=0$ when $\phi=0$. At the same time the noise disappears on both modes if $t=1$. This means that the full effect of the noise on the system becomes manifest only for $\phi>0$ and $t<1$, that is when the cavity modes are nonorthogonal. Assuming that noise operators belonging to left- and right-traveling modes do commute,

$$
\begin{aligned}
& {\left[\hat{f}_{\text {Lin }}, \hat{f}_{\text {Lin }}^{\dagger}\right]=1=\left[\hat{f}_{R \text { in }}, \hat{f}_{R \text { in }}^{\dagger}\right],} \\
& {\left[\hat{f}_{L \text { in }}, \hat{f}_{R \text { in }}^{\dagger}\right]=0=\left[\hat{f}_{R \text { in }}, \hat{f}_{L \text { in }}^{\dagger}\right],}
\end{aligned}
$$

we find that, in the general case $\phi \neq 0$ and $t \neq 1$, the noise operators $\hat{f}_{a}$ and $\hat{f}_{b}$ do not commute, 


$$
\begin{aligned}
& {\left[\hat{f}_{a}, \hat{f}_{a}^{\dagger}\right]=\left(1-t^{4}\right) \cos ^{2} \phi,} \\
& {\left[\hat{f}_{b}, \hat{f}_{b}^{\dagger}\right]=\left(1-t^{4}\right) \sin ^{2} \phi,} \\
& {\left[\hat{f}_{a}, \hat{f}_{b}^{\dagger}\right]=-\left(1-t^{4}\right) \sin \phi \cos \phi .}
\end{aligned}
$$

This noise correlation disappears when the modes become orthogonal $(\phi=0, \pi / 2$, and/or $t=1)$.

\section{B. Nonorthogonal modes and the Petermann excess noise factor}

Having found the relations between operators belonging to right-traveling and left-traveling modes after one round trip, we now show that our model effectively describes a cavity with nonorthogonal modes and can therefore show, in principle, excess quantum noise [8,37]. Although Eq. (32) has been written in a quantum context, it is equally valid in a classical context if one substitutes for the various operators $\hat{a}_{1 R}, \hat{b}_{1 R}$, etc. the corresponding classical complex amplitudes $\mathcal{A}_{1 R}, \mathcal{B}_{1 R}$, etc. and disregards the noise operators $\hat{f}_{a}$ and $\hat{f}_{b}$. The remaining homogeneous equation describes the round trip variation of a classical field inside the cavity. Furthermore, if one puts $G=1$ then the classical counterpart of Eq. (32) reduces to

$$
\left(\begin{array}{c}
\mathcal{A}_{1 R} \\
\mathcal{B}_{1 R}
\end{array}\right)=\mathbf{M}\left(\begin{array}{c}
\mathcal{A}_{1 L} \\
\mathcal{B}_{1 L}
\end{array}\right)
$$

where

$$
\mathbf{M} \equiv-\left(\begin{array}{cc}
\gamma_{+} \cos (2 \phi)+\gamma_{-} & \gamma_{+} \sin (2 \phi) \\
-\gamma_{+} \sin (2 \phi) & \gamma_{+} \cos (2 \phi)-\gamma_{-}
\end{array}\right),
$$

which coincides, apart from a multiplicative factor, with the classical cold cavity round-trip matrix $\mathbf{M}_{R T}$. Now, following Ref. [8], we find the eigenvalues and the eigenvectors of the matrix $\mathbf{M}$ and show that the latter ones form a nonorthogonal two-dimensional basis.

First we note that when $\phi=0$ or $t=1, \mathbf{M}_{R T}$ reduces to

$$
\phi=0 \Rightarrow \mathbf{M}=-\left(\begin{array}{ll}
t^{2} & 0 \\
0 & 1
\end{array}\right)
$$

or

$$
t=1 \Rightarrow \mathbf{M}=-\left(\begin{array}{cc}
\cos 2 \phi & \sin 2 \phi \\
-\sin 2 \phi & \cos 2 \phi
\end{array}\right)
$$

It is clear that in both these cases the eigenvectors are orthogonal. In the general case the eigenvalues $\lambda_{ \pm}$are

$$
\lambda_{ \pm}=\frac{-1}{2}\left[\left(1+t^{2}\right) \cos 2 \phi \pm Z\right]
$$

where

$$
Z \equiv\left[\left(1-t^{2}\right)^{2}-\left(1+t^{2}\right)^{2} \sin ^{2} 2 \phi\right]^{1 / 2}
$$
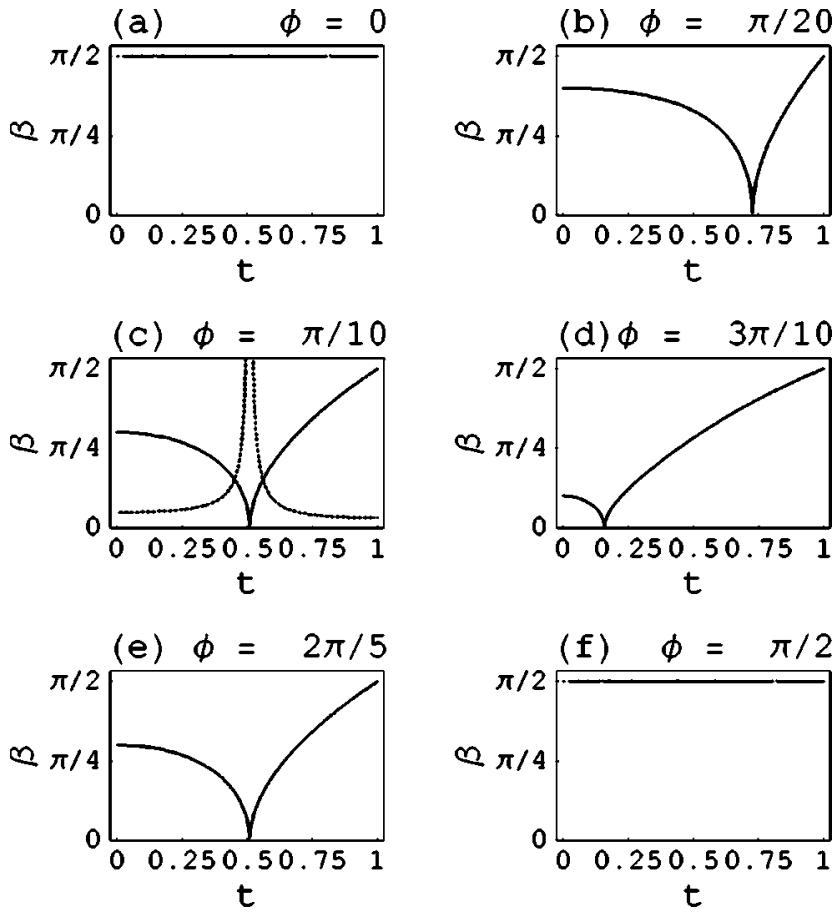

FIG. 2. (a)-(f) Angle $\beta$ between the cavity eigenmodes $\mathbf{u}_{ \pm}$versus the absorber parameter $t$ for different values of the rotator angle $\phi$. For $t=1$ the eigenmodes are always parallel $(\beta=\pi / 2)$ irrespective of the value of $\phi$. For $t=t_{c}(\phi)$ the eigenmodes become parallel: $\beta=0$. In (c) the dotted line gives the Petermann $K$ factor $(\times 1 / 50)$ as calculated from Eqs. (47) $-(48)$; the divergence appears for $t=t_{c}(\phi=\pi / 10) \approx 0.51$ when the cavity eigenmodes are parallel.

Depending on the values assumed for $\phi$ and $t$ we may have either $Z$ real or purely imaginary. In the latter case it is convenient to define

$$
Z \equiv i \zeta, \quad \zeta \equiv\left[\left(1+t^{2}\right)^{2} \sin ^{2} 2 \phi-\left(1-t^{2}\right)^{2}\right]^{1 / 2},
$$

where $\zeta$ is real. The critical value of $t$ for which $Z$ becomes purely imaginary is given by

$$
t_{c}(\phi)=\left[\frac{1-|\sin 2 \phi|}{1+|\sin 2 \phi|}\right]^{1 / 2} .
$$

For $t<t_{c}(\phi)$ both eigenvalues are real and the cavity eigenmodes are degenerate; this regime is usually referred to as the locked regime [9,38]. Conversely, for $t>t_{c}(\phi)$ the eigenvalues Eq. (41) acquire an imaginary part and the degeneracy between eigenmodes is removed (unlocked regime). Let $\mathbf{u}_{ \pm}$ be the non-normalized eigenvectors corresponding to $\lambda_{ \pm}$, respectively,

$$
\mathbf{u}_{ \pm}=\left(\begin{array}{c}
\left(1-t^{2}\right) \cos 2 \phi \pm Z \\
-\left(1+t^{2}\right) \sin 2 \phi
\end{array}\right) \text {. }
$$

For arbitrary values of $t$ and $\phi$ these eigenvectors are not orthogonal. This is shown in Fig. 2 where the angle $\beta$ between $\mathbf{u}_{+}$and $\mathbf{u}_{-}$is plotted as a function of $t$ for several values of $\phi$. For $t=1$ we have $\beta=\pi / 2$ (orthogonal modes) for all values of $\phi$, while for the critical $t=t_{c}(\phi)$ we see that 
$\beta=0$ and the modes become parallel. In Fig. 2(c) the "geometrical" Petermann $K$ factor for the cold cavity is plotted together with $\beta$. As Siegman remarked years ago $[7,14]$, the geometrical Petermann $K$ factor, as given below, is an intrinsic property of the cavity eigenmodes which has nothing to do with the gain medium inside the cavity. It can be calculated using the well known recipe [9]

$$
\frac{1}{K}=1-\frac{\left|\left(\mathbf{u}_{+}, \mathbf{u}_{-}\right)\right|^{2}}{\left(\mathbf{u}_{+}, \mathbf{u}_{+}\right)\left(\mathbf{u}_{-}, \mathbf{u}_{-}\right)},
$$

obtaining

$$
K_{<}=\frac{\left(1-t^{2}\right)^{2}}{\left(1-t^{2}\right)^{2}-\left(1+t^{2}\right)^{2} \sin ^{2} 2 \phi},
$$

for $t<t_{c}(\phi)$, and

$$
K_{>}=\frac{\left(1+t^{2}\right)^{2} \sin ^{2} 2 \phi}{\left(1+t^{2}\right)^{2} \sin ^{2} 2 \phi-\left(1-t^{2}\right)^{2}},
$$

for $t>t_{c}(\phi)$. Apart from notation these results agree with earlier works $[9,12]$. In the limit of small rotator angle $\phi$ $\ll 1$ we have $t_{c}(\phi) \simeq 1-2 \phi$ which is very close to 1 . If we define the dissipative coupling $\tau$ as $t=\exp (-2 \tau)(\tau \geqslant 0)$ one simply notices that in the limit of small $\phi$ and $\tau$, the behavior of $K$ near the critical value $t_{c}$ is given by

$$
\begin{gathered}
K_{<} \approx \frac{1}{1-\frac{\phi^{2}}{\tau^{2}}}, \\
K_{>} \approx \frac{1}{1-\frac{\tau^{2}}{\phi^{2}}},
\end{gathered}
$$

in agreement with Ref. [9].

\section{RESULTS AND DISCUSSION}

In this section we calculate the SPDC rate of the subthreshold OPO shown in Fig. 1 and study how it depends on the "nonorthogonality parameters" $t$ and $\phi$. Equations (24) together with Eqs. (32) can be straightforwardly solved to express "out" operators in terms of "in" operators; this is done explicitly in the Appendix. The resulting expressions are very cumbersome and it is not useful to write them explicitly. Their general form is

$$
\hat{a}_{\mathrm{out}}=\sum_{\alpha=a, b}\left(S_{1 \alpha} \hat{\alpha}_{\mathrm{in}}+S_{2 \alpha} \hat{\alpha}_{\mathrm{in}}^{\dagger}+S_{3 \alpha} \hat{f}_{\alpha}+S_{4 \alpha} \hat{f}_{\alpha}^{\dagger}\right),
$$

and similarly for mode $b$, where $S_{i \alpha}$ are complicated functions of $t, \phi, G, R$, and $\omega L / c$. From the above results we calculate the average photon number emitted in modes $a$ and $b$ :

$$
\bar{n}_{\alpha}=\left\langle\hat{\alpha}_{\text {out }}^{\dagger} \hat{\alpha}_{\text {out }}\right\rangle_{\text {vac }}(\alpha=a, b)
$$

where the subscript "vac" indicates that the quantum expectation value is calculated for the incoming vacuum field. When both the absorber and rotator are switched off (orthogonal-mode case) we find $\bar{n}_{a}=\bar{n}_{b} \equiv \bar{n}$, where

$$
\bar{n}=\left(G^{2}-1\right)\left[\frac{1-R}{1-2 G \sqrt{R} \cos (2 \omega L / c)+R}\right]^{2} .
$$

This result is in agreement with Eq. (16) in Ref. [31]. The term inside the square brackets, when calculated for $G=1$, coincides with the spontaneous emission modification factor $F$ [39], but in our case it is quadratic because of nonlinearity [40]. At resonance ( $L=m \pi c / \omega$, with $m$ integer), a divergence appears for $\bar{n}$ when $G=(1+R) /(2 \sqrt{R})>1$, corresponding to the threshold of oscillation [41]. However, we are interested only in the subthreshold case where a privileged lasing mode is not selected. The average photon numbers emitted on modes $a$ and $b$, evaluated at resonance, in the general case $\phi \neq 0$ and $t \neq 1$, are shown in Fig. 3. The values of the nonlinear gain and the mirror reflectivity are $G$ $=1.01$ and $R=0.2$, respectively, corresponding to a subthreshold OPO. The behavior with respect to the variable $\phi$ of $\bar{n}_{a}$ and $\bar{n}_{b}$, is quite similar for $t \approx 1$. When $t \rightarrow 0$, mode $a$ is increasingly suppressed and $\bar{n}_{a} \rightarrow 0$. In the same limit mode $b$ does not disappear but is reduced by a factor $\approx 3$. We report in Fig. 4 the total average photon number $\bar{N} \equiv \bar{n}_{b}$ $+\bar{n}_{a}$, evaluated at resonance, as a function of the absorber transmission coefficient $t$ and of the rotation angle $\phi$ due to the rotator. The nonlinear gain $G$ and the output mirror reflectivity $R$ have been chosen as $G=1.01, R=0.2$, so that subthreshold operation is achieved.

From Fig. 4 it is clear that the local maxima of $\bar{N}$, for the $t$ variable, are located on the curve $\phi=0$ which corresponds to a cavity with orthogonal modes. This curve constitutes the upper boundary of the gray band shown in Fig. 5. The other points in the gray band represent all possible values of $\bar{N}$, calculated with the same parameters as in Fig. 4, for cavities with nonorthogonal modes. All these points are below the curve corresponding to orthogonal modes; so we do not find any enhancement of the twin-photon rate under these conditions.

This may be compared with the behavior of the geometrical $K$ factor, as given by Eqs. (47) and (48). Figure 5 shows the behavior of this $K$ factor with respect to $\bar{N}$, as a function of the absorber transmission $t$. Both $K$ and $\bar{N}$ are evaluated for $\phi=\pi / 8$; furthermore, $\bar{N}$ is evaluated for $G=1.01$ and $R=0.2$. From a geometrical point of view, when $t=t_{c}$ the cavity eigenmodes become parallel and the corresponding $K$ factor diverges, as shown in Fig. 2(c). In Fig. 5 this resonant behavior of $K$, when $t$ approaches $t_{c}$, is evident, but at the same time there is no signature of a critical behavior of $\bar{N}$. Therefore we conclude that for a subthreshold OPO, the total average photon number $\bar{N}$ does not depend on $K$. 

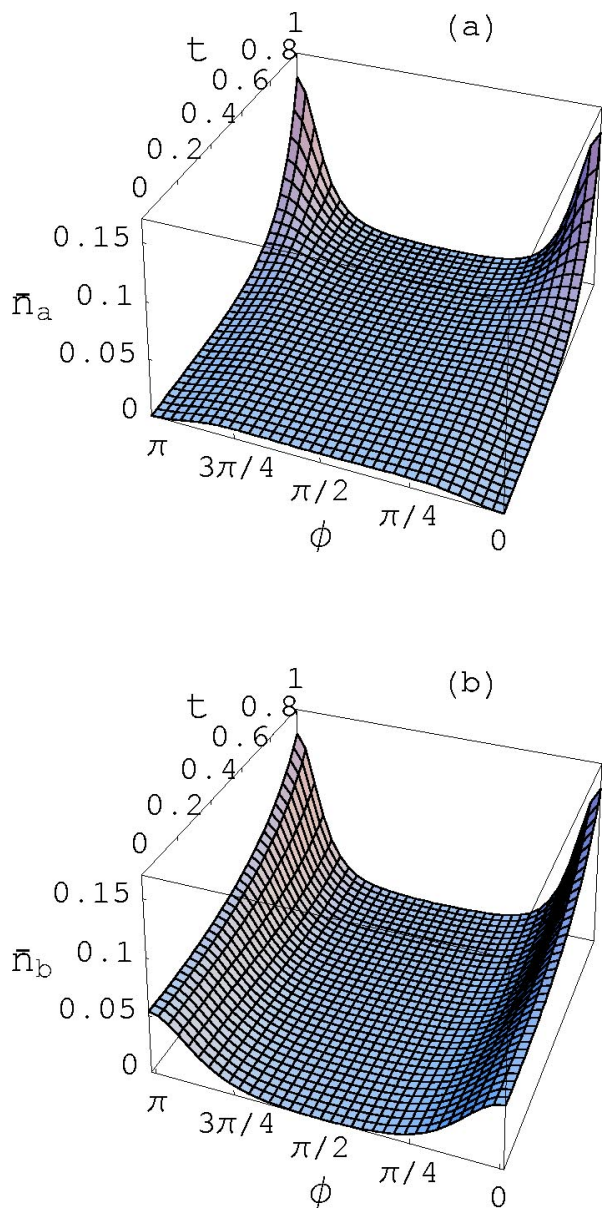

FIG. 3. (a) Plot of the average number $\bar{n}_{a}$ of photons emitted on mode $a$ for a subthreshold OPO at resonance as a function of the rotator angle $\phi$ and the absorber parameter $t$. The values of the other parameters are $G=1.01, R=0.2$. For $t=0$ and $\phi=0$ the photons in mode $a$ are fully absorbed so that $\bar{n}_{a}=0$. (b) Plot of the average number $\bar{n}_{b}$ of photons emitted in mode $b$ under the same conditions as in (a).

\section{OPTICAL BAND STRUCTURE IN A PARAMETRIC OSCILLATOR}

In the preceding section we have calculated the total average photon number $\bar{N}=\bar{n}_{a}+\bar{n}_{b}$ of the subthreshold OPO calculated at resonance, that is for $\omega L / c=m \pi$, where $m$ is an integer. In general the number $\bar{N}$ varies as a function of the phase shift $\theta \equiv \omega L / c$ which plays the role of a reduced length. It can be varied either by varying the length $L$ of the cavity or by varying the pump frequency $\Omega=2 \omega$. Then we can regard the function $\bar{N}(\theta)$ (calculated for fixed values of the other OPO parameters $t, G, R$, and $\phi$ ) as the cavity spectrum. In Fig. 6 we plot $\bar{N}$, calculated for $G=1.01$ and $R=0.2$ (below threshold $\mathrm{OPO}$ ), versus the length $\theta$ and the rotator angle $\phi$, for several values of the absorber parameter $t$. The function $\bar{N}(\theta, \phi)$ has, for $\phi=0$ and all values of $t$, the expected periodic behavior (with period $\pi$ ) which is characteristic of the spectrum of a Fabry-Pérot cavity. For decreasing $t$ the height of the resonant peaks is lowered but their

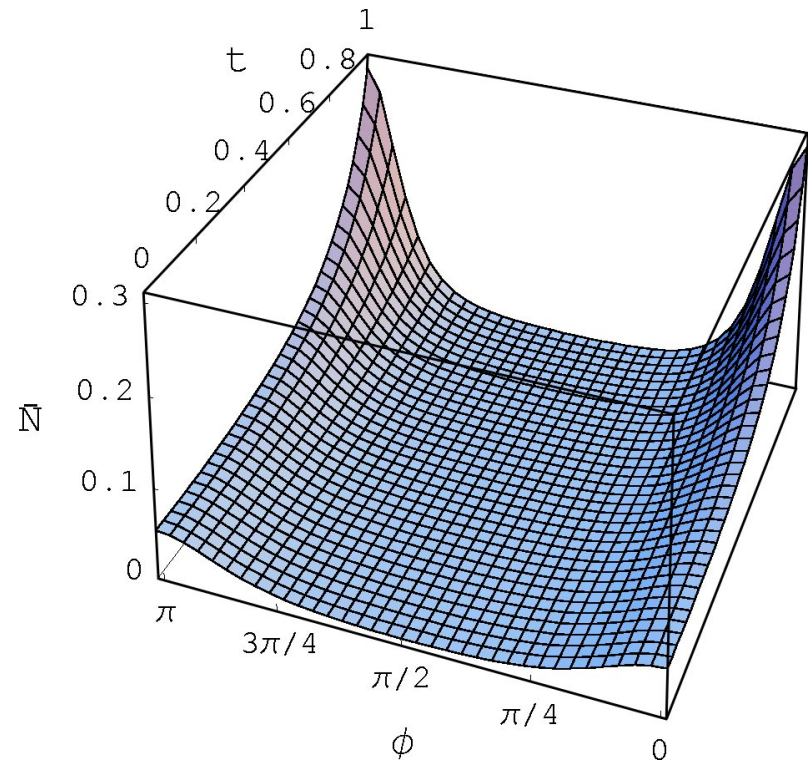

FIG. 4. Plot of the total average photon number $\bar{N} \equiv \bar{n}_{a}+\bar{n}_{b}$ of the subthreshold OPO, calculated at resonance, as a function of the absorber transmission $t$ and of the rotator angle $\phi$. The values of the other parameters are: $G=1.01, R=0.2$. For $t=0$ and $\phi=0$ the photons in mode $a$ are fully absorbed and the residual value of $\bar{N}$ is due to contribution of only mode $b$.

shape and position are unchanged. For $\phi=\pi / 2$ and all values of $t$ we obtain the same spectrum as for $\phi=0$ but shifted in the variable $\theta$ by an amount $\pi / 4$. This happens because $\phi$ $=\pi / 2$ simply corresponds, from a physical point of view, to an exchange of the role of the two orthogonal polarizations. For $\phi>0$ and $t>0$ each resonant peak is split in two separate bands corresponding to cavity eigenmodes with $y$ and $z$ polarization. The degeneracy is removed because of the polarization mode coupling induced by the rotator [see Eqs.

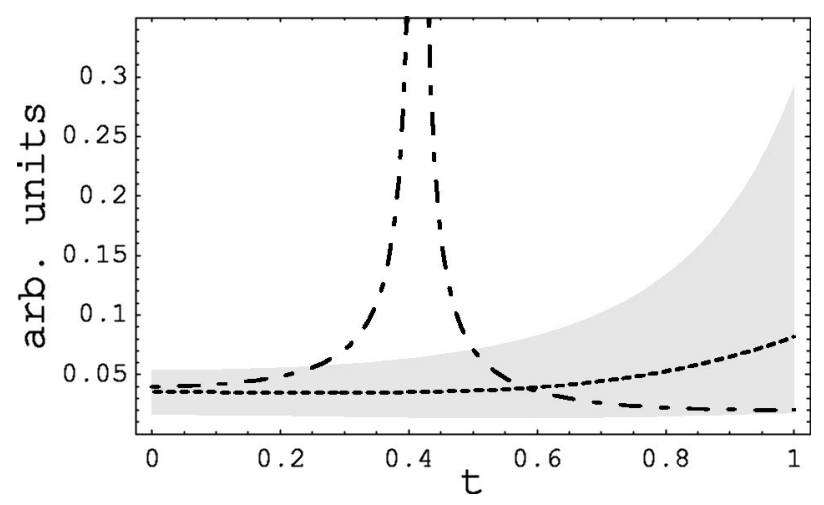

FIG. 5. Dotted-dashed line: "geometrical" Petermann $K$ factor, given by Eqs. (47) and (48) for a cavity without crystal, calculated for $\phi=\pi / 8$, as a function of the absorber transmission $t$. The value of $K$ diverges for $t \rightarrow t_{c}(\phi=\pi / 8) \simeq 0.41$. Dashed line: total average photon number $\bar{N}$ calculated at resonance and $\phi=\pi / 8$. The values of the other parameters are $G=1.01, R=0.2$, corresponding to a subthreshold OPO. The gray band represents all possible values of $\bar{N}$ for nonorthogonal modes. Note that $\bar{N}$ is not enhanced for $t=t_{c}$. 

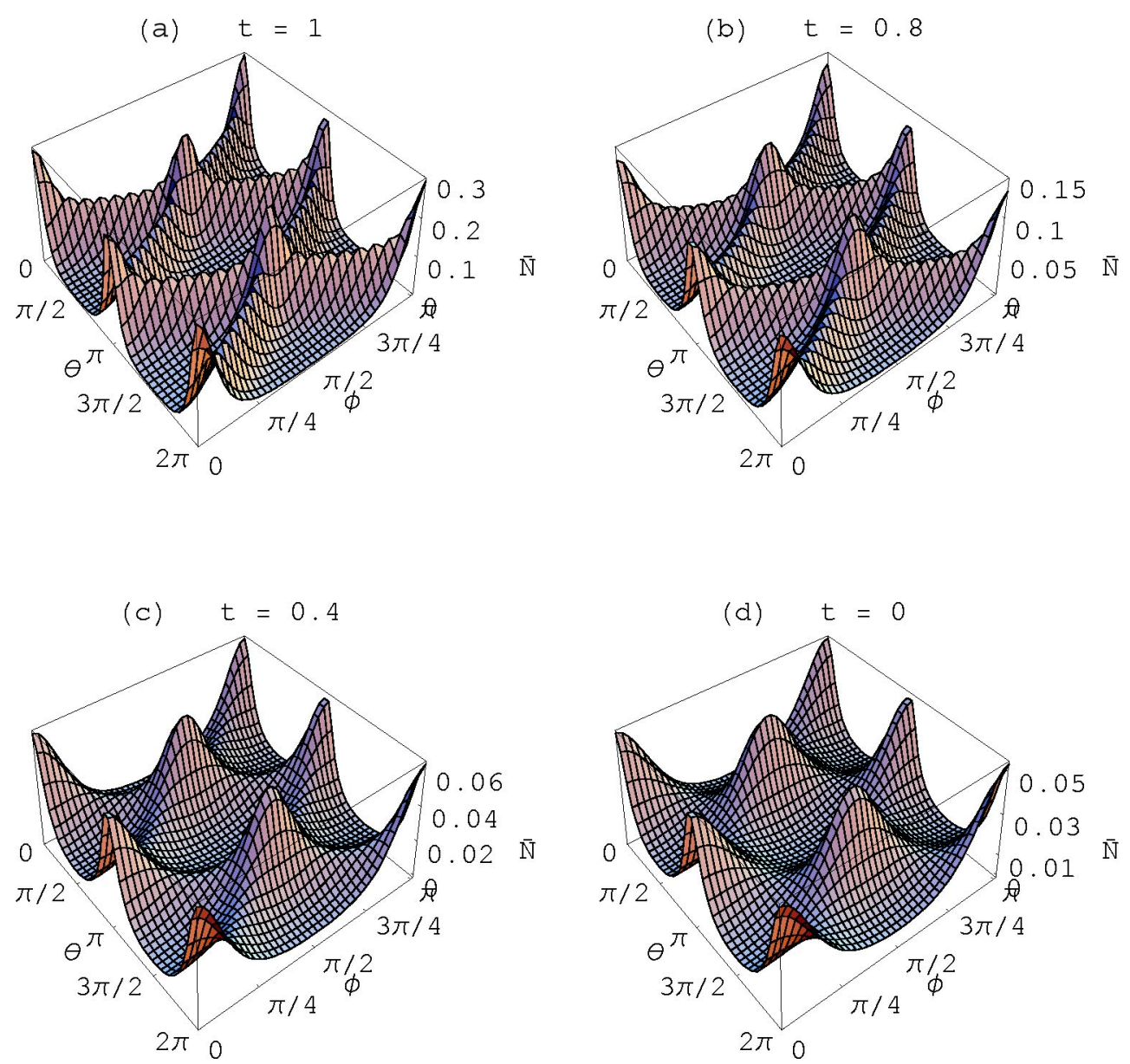

FIG. 6. Emission spectrum $\bar{N}(\theta, \phi)$ for a subthreshold OPO calculated for $G=1.01, R=0.2$, and different values of $t$. (a) For a cavity with orthogonal modes $(t=1)$ we have the periodic behavior characteristic of the spectrum of a Fabry-Pérot cavity, but only so for $\phi=0$ and $\phi=\pi / 2$. For other values of $\phi$ two resonant peaks for free spectral range appear. (b) and (c) For a cavity with nonorthogonal modes $(t$ $<1), \bar{N}(\theta, \phi)$ decreases with respect to the $t=1$ case but the doubling of the resonant peak remains.

(25)]. Finally, for $t=0$ there is an abrupt jump in the band structure for $\phi=\pi / 4$ because mode $a$ is totally suppressed and only a single linearly polarized mode can exist in the cavity. Actually this jump is not clearly visible in Fig. 6, but it becomes evident in Fig. 8.

The existence of optical band structures is well known for the case of a classical ring resonator, with passive polarization-optical elements $[32,42]$. In that case counterpropagating polarized waves are coupled by electro-optic modulators (EOM), Faraday rotators, partial reflectors, etc., that are arranged in a ring configuration. The polarizationmode eigenfrequencies then display band structures as a function of a tuning parameter, e.g., the voltage across an EOM. A general method for determining the eigenfrequency band structure in a ring cavity containing various passive optical elements, has been developed in Refs. [32,42]. Optical elements are represented by $4 \times 4$ matrices which couple two polarization degrees of freedom: $x$ and $y$ polarized waves, and two momentum degrees of freedom: clockwise (cw) and counterclockwise (ccw) waves. The spectrum of a ring cavity is determined by solving the secular equation for eigenvalue unity,

$$
\operatorname{det}\left(\mathbf{M}_{R T}-\mathbf{1}\right)=0
$$

where $\mathbf{M}_{R T}$ is the matrix for one round trip along the sequence: $\mathbf{M}_{R T}=\mathbf{M}_{n} \cdots \mathbf{M}_{2} \mathbf{M}_{1}$, and $\mathbf{M}_{1}, \mathbf{M}_{2}, \ldots$ are the individual optical element matrices.

This approach is inherently classical because it neglects the coupling between the cavity modes and the world outside the cavity. Since our OPO is inherently a quantum system which, moreover, is based upon a Fabry-Pérot cavity instead of a ring cavity, we have to be careful before adopting the same method. Equation (53) implicitly defines what is a spectral resonance for a classical ring cavity; we need an analogous definition in our quantum case. Input-output relations for a field inside a cavity with nonorthogonal modes were already discussed from a very general point of view by Grangier and Poizat [37]; however, their analysis concerned only a cavity with a linear medium inside. In our case we shall find that the classical equation (53) remains valid in the quantum context but acquires a different meaning.

\section{A. Resonance conditions: Quantum theory}

Now we extend to the quantum regime the treatment that leads to Eq. (53). The theory we have formulated in Sec. II 


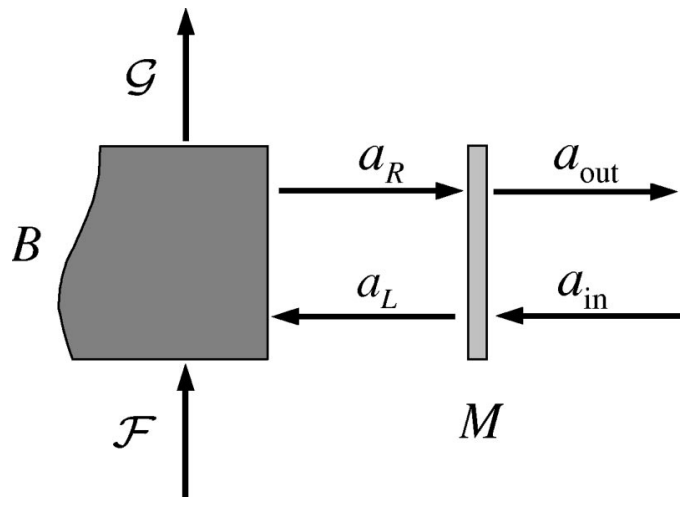

FIG. 7. Generalized one-output-mirror cavity. A mirror $M$ is put in front of a bulk material $B$. The sets of annihilation field operators inside and outside the cavity are written as $a_{R}, a_{L}$ and $a_{\text {out }}, a_{\text {in }}$, respectively. Analogously $\mathcal{F}(\mathcal{G})$ represents the set of annihilation input (output) noise operators.

can be generalized to an arbitrary linear amplifier (in the sense of Caves [1]) inside a cavity. A generalized one-outputmirror cavity is formed by a mirror $M$ put in front of a bulk material $B$ as shown in Fig. 7. Horizontal arrows represent field modes, that is modes of the electromagnetic both inside and outside the cavity. Vertical arrows represent noise modes, that is modes introduced to account for the loss channels. We denote the set of left-traveling field modes by $\mathcal{L}$ and the set of right-traveling field modes by $\mathcal{R}$ and assume $\operatorname{dim}(\mathcal{L})=\operatorname{dim}(\mathcal{R}) \equiv N$. The set of annihilation operators associated with the input and output field modes is denoted by $\mathbf{a}_{\text {in }}=\left[\left(a_{\text {in }}\right)_{1} \cdots\left(a_{\text {in }}\right)_{N}\left(a_{\text {in }}^{\dagger}\right)_{1} \cdots\left(a_{\text {in }}^{\dagger}\right)_{N}\right]^{T}$ and $\mathbf{a}_{\text {out }}$ $=\left[\left(a_{\text {out }}\right)_{1} \cdots\left(a_{\text {out }}\right)_{N}\left(a_{\text {out }}^{\dagger}\right)_{1} \cdots\left(a_{\text {out }}^{\dagger}\right)_{N}\right]^{T}$, respectively. The set of annihilation operators associated with the input noise modes is denoted by $\mathbf{f}=\left[(\mathcal{F})_{1} \cdots(\mathcal{F})_{N}\left(\mathcal{F}^{\dagger}\right)_{1} \cdots\left(\mathcal{F}^{\dagger}\right)_{N}\right]^{T}$. All operators belonging to the input (output) field modes commute with all operators (and their corresponding adjoints) belonging to the input (output) noise modes. As shown with more details in the Appendix, if we indicate with $\mathbf{R}$, T, and $\mathbf{M}$ three $2 N \times 2 N$ matrices which represent the reflectivity and transmittivity of the output mirror and the whole cavity, respectively, we find

$$
\begin{aligned}
\mathbf{a}_{\text {out }} & =(\mathbf{R}+\mathbf{T G T}) \mathbf{a}_{\text {in }}+\mathbf{T}(\mathbf{1}+\mathbf{G R}) \mathbf{f} \\
& \equiv \mathbf{S a}_{\text {in }}+\mathbf{F},
\end{aligned}
$$

where $\mathbf{G} \equiv \mathbf{M}(\mathbf{1}-\mathbf{R M})^{-1}$. Equation (54a) can be straightforwardly interpreted in term of transmitted and reflected field amplitudes, exactly as in the classical Fabry-Pérot interferometer theory. Looking at Eq. (54a) we see that the first term $\mathbf{R a}_{\text {in }}$ corresponds to the first reflected wave while the second term $\left(\mathbf{T G T a}_{\text {in }}\right)$ is the product of the wave coupled into the cavity which interacts with the optical elements represented by $\mathbf{G}$ and finally is coupled out of the cavity. In a similar manner we can interpret the noise term. Note that the solution Eq. (54) exists only if

$$
\operatorname{det}(\mathbf{1}-\mathbf{R M}) \neq 0 .
$$
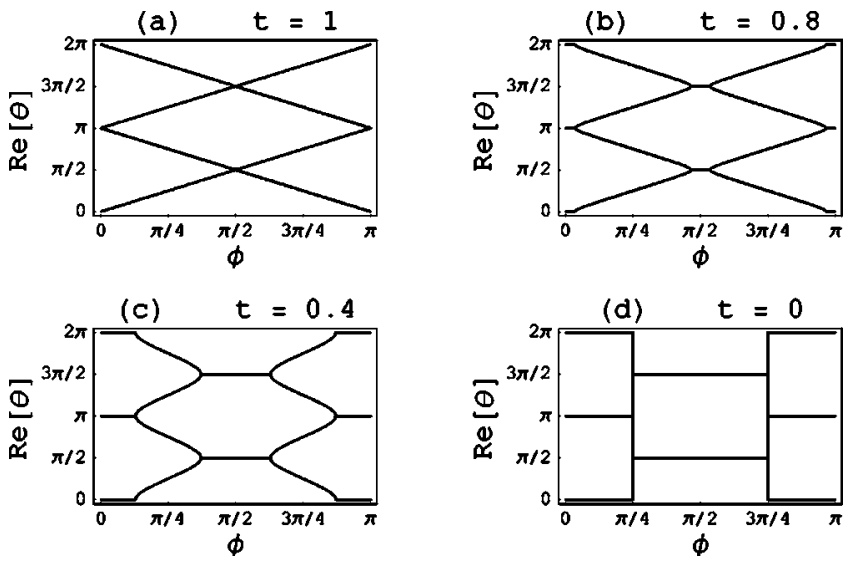

FIG. 8. Frequency band structures corresponding to the threedimensional spectra shown in Fig. 6. We have plotted $\operatorname{Re}\left(\theta_{\text {res }}\right)$ versus the rotator angle $\phi$ for different values of $t$.

Now we are ready to reexamine the definition of a spectral resonance. From the general equation (54) it is clear, by inspection, that all $S$-matrix elements have a common denominator $D(\theta)(\theta \equiv \omega L / c)$ equal to $D(\theta)=\operatorname{det}(\mathbf{1}-\mathbf{R M})$. A natural definition of the resonant values $\theta_{\text {res }}$ is then given by the complex zeros of $D(\theta)$ [43]. From a physical point of view, since $\omega$ and $L$ are real variables, we consider $\operatorname{Re}\left(\theta_{\text {res }}\right)$ as the true resonant frequency. With this definition $D\left(\operatorname{Re}\left(\theta_{\text {res }}\right)\right) \neq 0$ and our previous calculations apply. As an example of this definition we show in Fig. 8 the frequency band structure corresponding to the spectra already shown in Fig. $6 . \operatorname{Re}\left(\theta_{\text {res }}\right)$ is plotted versus the rotator angle $\phi$ for different values of $t$. When $\phi=0$ the two modes corresponding to polarizations $a$ and $b$ are degenerate in frequency for all values of $t$. This degeneracy is removed by the rotator which induces a coupling between the two polarization modes. When $\phi=\pi / 2 \bmod (2 \pi)$ the two modes exchange their role and the spectrum is simply shifted by $\pi / 2$. For $t$ $=0$ the polarization mode $a$ is completely suppressed and the spectrum is again degenerate.

We now return to our discussion of the resonance condition to notice that, when $\operatorname{Im}\left(\theta_{\text {res }}\right)=0$, the determinant is zero for real frequencies and our calculations break down. However, the real solutions of the equation $D(\theta)=0$ constitute a set of functions $\theta_{i}(R, G, \phi, t)(i=1,2, \ldots)$, which fix the boundary of the domain, in the space of the parameters $R, G$, $\phi$, and $t$, within which solutions of Eq. (54a) exist. In fact it is clear that, being $\theta \propto \omega \propto k$, the solutions, in general complexes, of the equation $D(\theta)=0$ are the analog of the circle of convergence of the geometrical series $s(z)$ in the FabryPérot transmission function. In the classical theory of the Fabry-Pérot interferometer a plane wave impinging on one of the mirrors of the interferometer is partially transmitted and partially reflected. The amplitude of both the transmitted and reflected wave is proportional to the sum of a geometrical series $s(z)=1+z+z^{2}+\cdots$ whose argument is $z$ $=r e^{i 2(k L+\phi)}$ for normal incidence. This series can be summed only if $|z|<1$. In our case $|z|=r e^{-2 k_{i} L}$ which is less than 1 only if $k_{i}>k_{i}^{\text {th }}$ were 

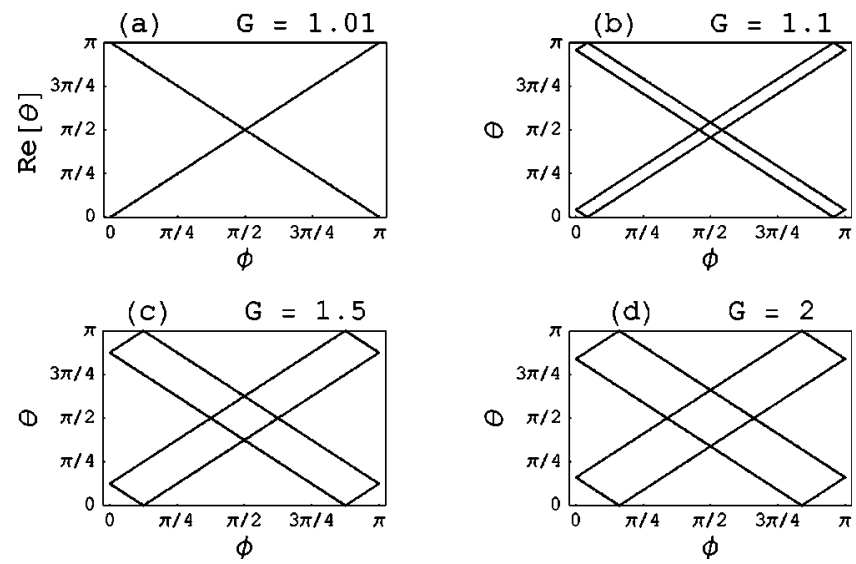

FIG. 9. Illustrating the doubling mechanism for an OPO in a cavity with orthogonal eigenmodes $(t=1)$ and mirror reflectivity $R=0.5$. For increasing values of $G$ the gap between bands is also increasing. Higher values of $G$ are not considered here because our model is limited by the nondepleted pump approximation.

$$
k_{i}^{\mathrm{th}}=-\frac{1}{2 L} \ln \left(\frac{1}{r}\right) .
$$

It is clear that the threshold condition corresponds to a value of $z=x+i y$ which lies, in the complex plane $(x, y)$, exactly on the radius of convergence of the geometrical series $S(z)$. Analogously we identify the points lying on the boundary functions $\theta_{i}(R, G, \phi, t)$ with the set of the values of the parameters $R, G, \phi$, and $t$ for which oscillations start (threshold values) and therefore we write the threshold condition as $\operatorname{Im}\left(\theta_{\text {res }}\right)=0$. In the next section we analyze the distribution of these singular points in the plane $(\theta, \phi)$ for different values of $R, G$, and $t$. Finally it is interesting to note that the "quantum" equation $D(\theta)=\operatorname{det}(\mathbf{1}-\mathbf{R M})=0$ does not contain any noise contribution and is, in fact, completely classical and therefore fully equivalent to the "classical" equation (53).

\section{B. Mode spectra of OPO}

We start our analysis of the OPO optical band structure by considering what happens in a cavity with orthogonal modes $(t=1)$ when the threshold condition $\operatorname{Im}\left(\theta_{\text {res }}\right)=0$ is satisfied. In Fig. 9 the frequency band structure of the OPO spectrum is plotted for increasing values of the gain $G$ and fixed mirror reflectivity $R=0.5$. For $G=1.01$ (subthreshold OPO) we have $\operatorname{Im}\left(\theta_{\text {res }}\right) \neq 0$ and the spectrum is the same as Fig. 8(a). For $G>G_{\mathrm{th}}(R) \equiv(1+R) /(2 \sqrt{R})$ each band is doubled and shifted and the gap between two near bands is increasing along with $G$. In other words a degeneracy between two eigenmodes is removed when the OPO starts to oscillate. At first sight these band structures closely resemble the corresponding ones in passive ring cavities [see, e.g., Fig. 2(d) in [32]]. In a ring cavity the doubling in the band structure arises from the coupling between counterpropagating modes along the ring. In other words, in a ring cavity the four degrees of freedom of the electromagnetic field that are responsible for the presence of four resonant peaks per free spectral range are two "polarization" degrees of freedom and two
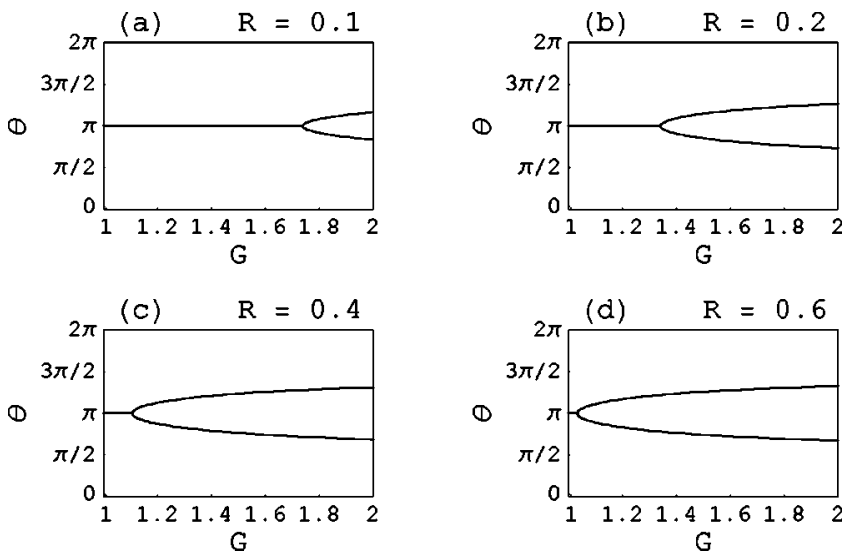

FIG. 10. Illustrating the bifurcation appearing around a single resonant peak in the OPO spectrum in a Fabry-Pérot cavity with orthogonal eigenmodes ( $\phi=0$ and $t=1)$. When increasing the mirror reflectivity $R$ the system approaches the threshold of oscillation for smaller values of $G$.

"momentum" degrees of freedom. Instead in our case the doubling is due to the coupling between annihilation and creation operators belonging to different polarization modes. In other words here we have two polarization degrees of freedom coupled in a linear way by passive optical devices, and the same two polarization degrees of freedom coupled in a nonlinear way by the crystal. To see this more clearly, the band structure for an OPO in a simple linear cavity $(t=1$ and $\phi=0$ ) is shown in Fig. 10, where $\theta_{\text {res }}$ is plotted versus $G$ for different values of $R$. When $G$ approach the threshold value $G_{\mathrm{th}}(R)$, a bifurcation in the OPO spectrum appears.

This bifurcation should be, in principle, observable experimentally. However, the well-known instability of a near threshold OPO [41], which is perhaps connected with this bifurcation, could make its direct observation very difficult. However, a detailed analysis of the OPO instability and its connection with the spectrum bifurcation other than with self-phase-locking (see, e.g., [44]) goes beyond the scopes of the present work. We simply recall that in our calculation the crystal is considered infinitesimally thin so that the bifurcation cannot be explained as a refractive index-dependent propagation effect within the crystal. The true nature of this phenomenon lies in the nonlinear coupling due to the crystal between annihilation and creation operators belonging to different polarization modes, as is made clear in Fig. 9. In order to understand this in detail, we rewrite the scattering matrix of a parametric amplifier as

$$
\begin{aligned}
& b_{1}=\cosh \gamma a_{1}+\sinh \gamma a_{2}^{\dagger}, \\
& b_{2}=\cosh \gamma a_{2}+\sinh \gamma a_{1}^{\dagger},
\end{aligned}
$$

where $G=\cosh \gamma$. Ou [45] has shown that under the transformations

$$
\begin{aligned}
& a_{ \pm}=\left(a_{1} e^{-i \delta} \pm a_{2} e^{i \delta}\right) / \sqrt{2}, \\
& b_{ \pm}=\left(b_{1} e^{-i \delta} \pm b_{2} e^{i \delta}\right) / \sqrt{2},
\end{aligned}
$$



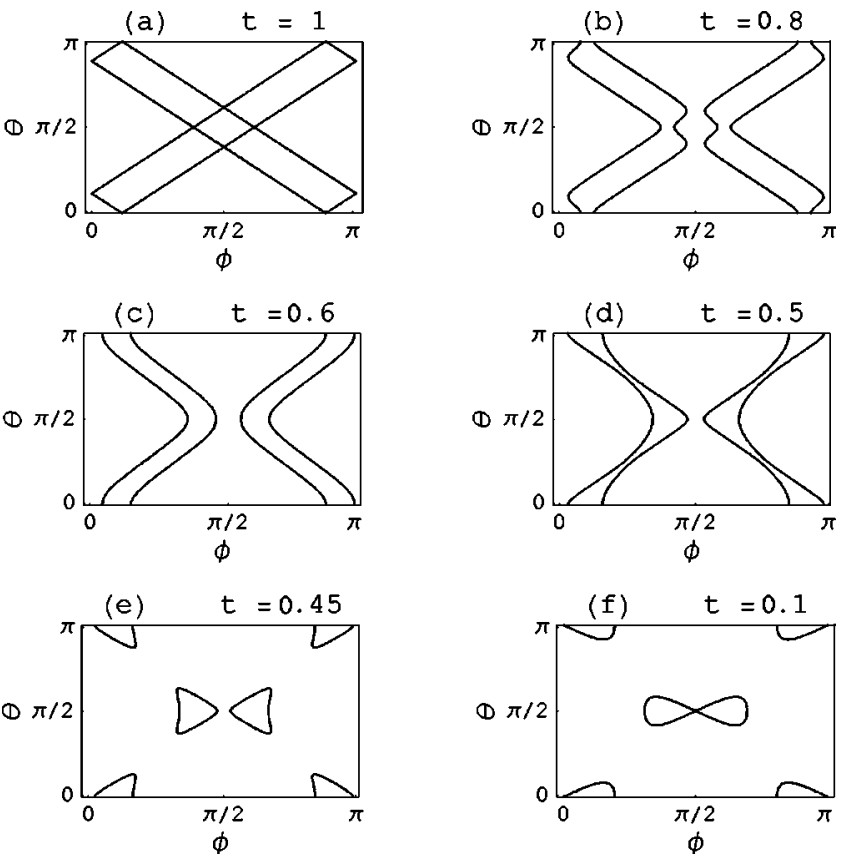

FIG. 11. Frequency band structures of an OPO in a cavity with nonorthogonal eigenmodes. The cavity "length" $\theta=\omega L / c$ is plotted versus the rotator angle $\phi$ for several values of the absorber parameter $t$. The values of the other parameters are $G=\sqrt{2}, R$ $=0.5$.

( $\forall \delta \in \mathbb{R}$ ) Eqs. (57) decouple in the equations of two independent degenerate parametric amplifiers,

$$
\begin{aligned}
& b_{+}=\cosh \gamma a_{+}+\sinh \gamma a_{+}^{\dagger}, \\
& b_{-}=\cosh \gamma a_{-}-\sinh \gamma a_{-}^{\dagger} .
\end{aligned}
$$

A degenerate parametric amplifier is, following the definition of Caves [1], a phase-sensitive amplifier, that is an amplifier which responds differently to the two quadrature phases of the field defined as

$$
\begin{gathered}
q(a)=\frac{a+a^{\dagger}}{\sqrt{2}}, \\
p(a)=\frac{a-a^{\dagger}}{\sqrt{2} i} .
\end{gathered}
$$

These operators are both Hermitian and thus, in principle, observable. From Eqs. (59), (60) it is easy to see that each quadrature phase is amplified with a different gain,

$$
\begin{aligned}
& q_{+}(b)=e^{\gamma} q_{+}(a), \quad p_{+}(b)=e^{-\gamma} p_{+}(a), \\
& q_{-}(b)=e^{-\gamma} q_{-}(a), \quad p_{-}(b)=e^{\gamma} p_{-}(a),
\end{aligned}
$$

and we have four independent observable degrees of freedom. In fact, from Eqs. (61), (62), we see that only two quadrature phases really exhibit different gain. Since the threshold condition $\operatorname{Im}\left(\theta_{\text {res }}\right)=0$ contains explicitly the gain,
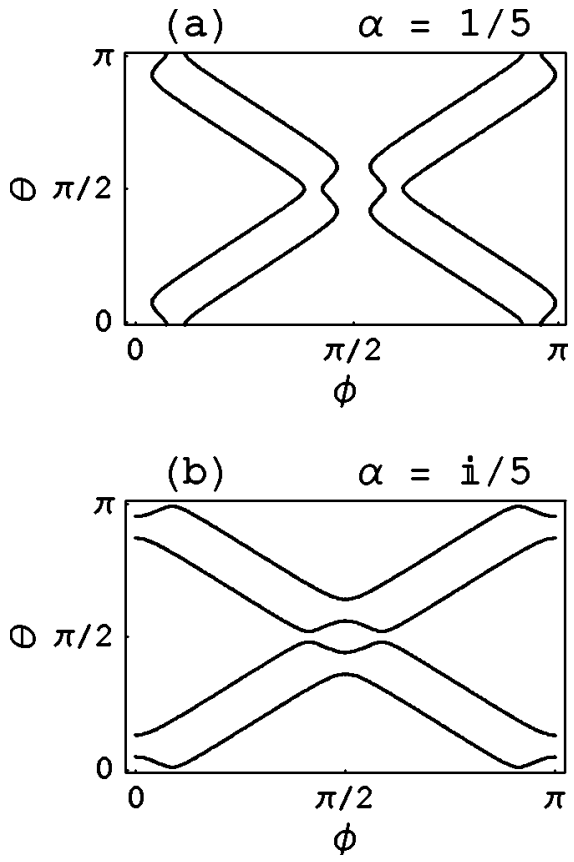

FIG. 12. Illustrating the effect of nonunitarity on the frequency band structure. Two band structures are shown, calculated for an OPO with $G=\sqrt{2}, \quad R=0.5$ and (a) $t=\exp (-1 / 5)$ and (b) $t=\exp$ $(-i / 5)$. The two band structures have the same shape but they differ by a $\pi / 2$ rotation in the plane of the figure.

each of these two quadrature phases reaches the threshold for a different set of values of the parameters $R, G, \phi$, and $t$ and two bands appear in the spectrum.

Now we consider the more general case of a cavity with nonorthogonal modes $t \leqslant 1$ and $\phi \neq 0$. In this case all four quadrature phases are coupled to each other and four bands appear. The frequency band structure of the OPO spectrum is shown in Fig. 11 where $\theta_{\text {res }}$ is plotted versus the rotator angle $\phi$ for different values of $t$ and fixed $G=\sqrt{2}$ and $R=0.5$. The difference between these spectra and the ones usually obtained for lossless ring cavities is both in the shape of the bands and also in their disposal. In our case the bands are symmetric with respect to a vertical axis while in the passive-cavity case the symmetry is with respect to a horizontal axis. This is clearly illustrated in Fig. 12 where the two pictures differ for a rotation by a $\pi / 2$ angle in the plane of the figure. This phenomenon is entirely due to the losses in our model represented by a nonunitary matrix. However, we stress the fact that this lack of unitarity only appears in the classical equation $\operatorname{det}(\mathbf{1}-\mathbf{R M})=0$ but not in the full quantum equation (54).

To illustrate this phenomenon we consider, for simplicity, a two-mode optical system which contains an absorbing element whose matrix can be written as [46]

$$
A=\left(\begin{array}{cc}
e^{-\alpha} & 0 \\
0 & e^{\alpha}
\end{array}\right)
$$

where $\alpha$ is a real parameter. This matrix is trivially nonunitary. Let us now analytically continue the real parameter $\alpha$ in 
the complex space via the transformation $\alpha \rightarrow \alpha e^{i \eta}$. After this transformation the matrix $A$ becomes

$$
A(\eta)=\left(\begin{array}{cc}
e^{-\alpha \cos \eta-i \alpha \sin \eta} & 0 \\
0 & e^{\alpha \cos \eta+i \alpha \sin \eta}
\end{array}\right),
$$

which is in general nonunitary for arbitrary values of the real parameter $\eta$. When $\eta=0$ we recover the original matrix $A$, while for $\eta=\pi / 2$ we obtain

$$
A(\pi / 2)=\left(\begin{array}{cc}
e^{-i \alpha} & 0 \\
0 & e^{i \alpha}
\end{array}\right),
$$

which is unitary. In Fig. 12 we show the effects of the transformation $\alpha \rightarrow \alpha e^{i \eta}$ on a eigenfrequency band structure: Fig. 12(b) is obtained from Fig. 12(a) by writing the absorber parameter as $t=\exp (-\alpha)$ and by making the substitution $\alpha$ $\rightarrow i \alpha$ in the boundary functions $\theta_{i}\left(R, G, \phi, t=e^{-\alpha}\right)$ introduced in the preceding section. Looking at Eq. (10) it is clear that the trick $\alpha \rightarrow \alpha e^{i \eta}$ can be interpreted as a "Wick rotation" [29] if one thinks of the parameter $\alpha$ as proportional to a finite time interval.

The nonunitary nature of the $\mathbf{M}$ matrix is also responsible for the lack of continuity in the band structure for values of $t$ less then 1, as shown in Figs. 11(e) and 11(f). The breaking of the band structures and the appearance of "islands" is due to the fact that when $t<1$ one polarization mode (mode $a$ in the preceding sections) is increasingly suppressed because of the losses introduced by the absorber. Since in Fig. 11 the assigned value of the gain $G$ corresponds to a near-threshold value $G_{\text {th }}$ only for $t=1$, when $t<1$ the increasing losses cause an increasing value of $G_{\text {th }}$ and some eigenmodes cannot start to oscillate. Particularly, for $t=0$, one mode is completely suppressed and only two resonant peaks (instead of four) per free spectral range are left. By explicit calculation it is easy to see that when using optical devices represented by a unitary matrix this phenomenon does not appear; this mode suppression can be achieved only using nonunitary optical devices.

\section{CONCLUSIONS}

In the first part of this paper we have introduced and analyzed a model for an optical parametric oscillator in a cavity with nonorthogonal polarization modes. Our model comprises (and reduces to those as particular subcases) two theoretical models both of which have been experimentally verified. For the type-II degenerate parametric amplifier we use the model of Gardiner and Savage [31] whose validity has recently been verified experimentally by $\mathrm{Ou}$ and $\mathrm{Lu}$ [23]. For the cavity with two nonorthogonal polarization modes, where large polarization $K$ factors have been demonstrated [9,12], we adopt the model of van der Lee et al. [9]. By using this model we have shown that there is no excess quantum noise enhancement in type-II SPDC. On the contrary, the use of a cavity with nonorthogonal (instead of orthogonal) eigenmodes leads to a reduction of the twin photon generation rate. Excess quantum noise must therefore be exclusively ascribed to amplification of spontaneously emitted photons; the spontaneous emission process itself is not affected. Excess quantum noise becomes effective only very close to threshold when one of the cavity eigenmodes is "selected" as the oscillating mode which dominates over the other modes [47].

In the second part of this paper we have studied the eigenfrequency spectrum of the same OPO, but now working near threshold. In order to find the correct definition of a spectral resonance within our fully quantum treatment, we have derived the spectral dependence of this resonance from the OPO parameters by writing explicitly the scattering matrix for the whole cavity. Since a type-II parametric crystal couples annihilation operators belonging to a certain polarization mode with creation operators belonging to the orthogonal polarization mode, we deal with a system which has four coupled degrees of freedom. Thus we have found that in the OPO spectrum four resonant peaks per free spectral range can exist. The "position" $\theta=\omega L / c$ of these peaks depends on the transmission $t$ of the absorber and on the rotator angle $\phi$ which also fix the "degree of nonorthogonality" of the cavity. Because of the $\phi$ dependence, different band structures, whose shapes depend on $t$, appear in the OPO spectrum. Since we are considering a degenerate parametric amplifier with $\omega=\Omega / 2$, in order to experimentally detect the spectral band structures we can either scan the cavity length $L$ or vary the pump frequency $\Omega$. These band structures closely resemble those found for a passive classical ring cavity [32].

\section{ACKNOWLEDGMENTS}

We acknowledge support from the EU under the ISTATESIT contract. This project is also supported by FOM.

\section{APPENDIX}

In this appendix we derive explicitly Eq. (54) utilized in Sec. V A. Let us consider the arrangement shown in Fig. 9. Horizontal arrows represent field modes, that is modes of the electromagnetic both inside and outside the cavity. Vertical arrows represent noise modes, that is modes introduced to account for the loss channels. We denote the set of lefttraveling field modes by $\mathcal{L}$ and the set of right-traveling field modes by $\mathcal{R}$ and assume $\operatorname{dim}(\mathcal{L})=\operatorname{dim}(\mathcal{R}) \equiv N$. The set of annihilation operators associated with the input (output) field modes is denoted by $a_{\text {in }}\left(a_{\text {out }}\right)$. The set of annihilation operators associated with the input (output) noise modes is denoted by $\mathcal{F}(\mathcal{G})$. All operators belonging to the input (output) field modes commute with all operators (and their corresponding adjoints) belonging to the input (output) noise modes. Finally we denote with $a_{L}$ and $a_{R}$ the set of operators belonging to the field modes inside the cavity. They satisfy the quite general linear relation

$$
\left(a_{R}\right)_{\alpha}=\sum_{\beta=1}^{N}\left[M_{\alpha \beta}\left(a_{L}\right)_{\beta}+L_{\alpha \beta}\left(a_{L}^{\dagger}\right)_{\beta}\right]+\mathcal{F}_{\alpha},
$$

where $\alpha=1, \ldots, N$. The $2 N^{2}$ complex numbers $M_{\alpha \beta}$ and $L_{\alpha \beta}$ are completely determined by the optical elements in- 
side the cavity. Even if each of these elements is represented by a unitary operator, the requirement that the operators in the sets $a_{R}$ and $a_{L}$ obey the bosonic commutation relations does not need to be satisfied since these operators are associated with intracavity modes [48].

The mirror $M$ generates a linear coupling between operators belonging to the sets $a_{L}, a_{R}, a_{\text {in }}, a_{\text {out }}$ which can be represented as

$$
\begin{aligned}
\left(a_{\text {out }}\right)_{\alpha} & =\sum_{\beta=1}^{N}\left[T_{\alpha \beta}\left(a_{R}\right)_{\beta}+R_{\alpha \beta}\left(a_{\text {in }}\right)_{\beta}\right], \\
\left(a_{L}\right)_{\alpha} & =\sum_{\beta=1}^{N}\left[T_{\alpha \beta}\left(a_{\text {in }}\right)_{\beta}+R_{\alpha \beta}\left(a_{R}\right)_{\beta}\right],
\end{aligned}
$$

$(\alpha=1, \ldots, N)$. Is is easy to solve Eqs. (A1) and (A2) together in order to express the operators $a_{\text {out }}$ as linear combinations of the operators $a_{\text {in }}$ and $\mathcal{F}$ (and their respective adjoints), as we have already done in Sec. I. However, in order to illustrate the nature of the solution that we have found and to show how the resonance condition can be imposed in a quantum theory, we solve again Eqs. (A1) and (A2) introducing a matrix notation. Let $\underline{M}$ stand for $M_{\alpha \beta}, \underline{L}$ for $L_{\alpha \beta}, \underline{T}$ for $T_{\alpha \beta}$ and $\underline{R}$ for $R_{\alpha \beta}$. All these are $N \times N$ matrices. With $a_{L}, a_{\text {in }}$, etc., now we indicate the $N$-component vectors $a_{L}=\left[\left(a_{L}\right)_{1}\left(a_{L}\right)_{2} \cdots\left(a_{L}\right)_{N}\right]^{T}, a_{\text {in }}=\left[\left(a_{\text {in }}\right)_{1}\left(a_{\text {in }}\right)_{2} \cdots\left(a_{\text {in }}\right)_{N}\right]^{T}$, etc., respectively and similarly for the corresponding adjoint operators. Using this notation we rewrite Eqs. (A1) as

$$
\left(\begin{array}{c}
a_{R} \\
a_{R}^{\dagger}
\end{array}\right)=\left(\begin{array}{cc}
\underline{M} & \underline{L} \\
\underline{L}^{*} & \underline{M}^{*}
\end{array}\right)\left(\begin{array}{c}
a_{L} \\
a_{L}^{\dagger}
\end{array}\right)+\left(\begin{array}{c}
\mathcal{F} \\
\mathcal{F}^{\dagger}
\end{array}\right)
$$

and Eqs. (A2) as

$$
\begin{aligned}
& \left(\begin{array}{c}
a_{\text {out }} \\
a_{\mathrm{out}}^{\dagger}
\end{array}\right)=\left(\begin{array}{cc}
\frac{T}{0} & 0 \\
0 & \underline{T}^{*}
\end{array}\right)\left(\begin{array}{c}
a_{R} \\
a_{R}^{\dagger}
\end{array}\right)+\left(\begin{array}{cc}
\underline{R} & 0 \\
0 & \underline{R}^{*}
\end{array}\right)\left(\begin{array}{c}
a_{\mathrm{in}} \\
a_{\mathrm{in}}^{\dagger}
\end{array}\right), \\
& \left(\begin{array}{c}
a_{L} \\
a_{L}^{\dagger}
\end{array}\right)=\left(\begin{array}{cc}
\frac{T}{0} & 0 \\
0 & \underline{T}^{*}
\end{array}\right)\left(\begin{array}{c}
a_{\mathrm{in}} \\
a_{\mathrm{in}}^{\dagger}
\end{array}\right)+\left(\begin{array}{cc}
\underline{R} & 0 \\
0 & \underline{R}^{*}
\end{array}\right)\left(\begin{array}{c}
a_{R} \\
a_{R}^{\dagger}
\end{array}\right) .
\end{aligned}
$$

This is only an intermediate step. We go ahead further introducing the $2 N$-component vectors $\mathbf{a}_{R}=\left(a_{R} a_{R}^{\dagger}\right)^{T}$, f $=\left(\mathcal{F} \mathcal{F}^{\dagger}\right)^{T}$, etc., and the $2 N \times 2 N$ matrices

$$
\mathbf{M}=\left(\begin{array}{cc}
\frac{M}{L} & \underline{L} \\
\underline{L}^{*} & \underline{M}^{*}
\end{array}\right)
$$

and

$$
\mathbf{T}=\left(\begin{array}{cc}
\frac{T}{2} & 0 \\
0 & \underline{T}^{*}
\end{array}\right), \quad \mathbf{R}=\left(\begin{array}{cc}
\underline{R} & 0 \\
0 & \underline{R}^{*}
\end{array}\right) .
$$

Now we are ready to rewrite Eqs. (A3) and (A4) as

$$
\begin{aligned}
& \mathbf{a}_{R}=\mathrm{Ma}_{L}+\mathbf{f}, \\
& \mathbf{a}_{\text {out }}=\mathbf{T a}_{R}+\mathbf{R a}_{\text {in }} \text {, } \\
& \mathbf{a}_{L}=\mathbf{T a} \mathbf{a}_{\text {in }}+\mathbf{R} \mathbf{a}_{R} .
\end{aligned}
$$

Inserting Eq. (A7a) in Eq. (A7c), solving for $\mathbf{a}_{L}$ and using this result in Eqs. (A7a) and (A7b) we finally find, for the operators belonging to the output field modes,

$$
\begin{aligned}
\mathbf{a}_{\text {out }} & =(\mathbf{R}+\mathbf{T G T}) \mathbf{a}_{\text {in }}+\mathbf{T}(\mathbf{1}+\mathbf{G R}) \mathbf{f} \\
& \equiv \mathbf{S a}_{\text {in }}+\mathbf{F},
\end{aligned}
$$

where $\mathbf{G} \equiv \mathbf{M}(\mathbf{1}-\mathbf{R} \mathbf{M})^{-1}$.
[1] C. M. Caves, Phys. Rev. D 26, 1817 (1982).

[2] A. E. Siegman, Lasers (University Science Books, Mill Valley, CA, 1996).

[3] A. Yariv, Quantum Electronics, 3rd ed. (John Wiley \& Sons, New York, 1988).

[4] J. P. Woerdman, M. P. van Exter, and N. J. van Druten, in Advances in Atomic, Molecular, and Optical Physics, edited by B. Bederson and H. Walther (Academic Press, Orlando, FL, 2001), Vol. 47.

[5] H. Yokoyama and K. Ujihara, Spontaneous Emission and Laser Oscillation in Microcavities, Laser and Optical Science and Technology (CRC Press, Boca Raton, 1995).

[6] K. Petermann, IEEE J. Quantum Electron. QE-15, 566 (1979).

[7] A. E. Siegman, Phys. Rev. A 39, 1253 (1989).

[8] P. Grangier and J.-P. Poizat, Eur. Phys. J. D 1, 97 (1998).

[9] A. M. van der Lee, N. J. van Druten, A. L. Mieremet, M. A. van Eijkelenborg, A. M. Lindberg, M. P. van Exter, and J. P. Woerdman, Phys. Rev. Lett. 79, 4357 (1997).

[10] W. A. Hamel and J. P. Woerdman, Phys. Rev. Lett. 64, 1506 (1990)

[11] Y.-J. Cheng, C. G. Fanning, and A. E. Siegman, Phys. Rev. Lett. 77, 627 (1996).
[12] O. Emile, M. Brunel, A. L. Floch, and F. Bretenaker, Europhys. Lett. 43, 153 (1998).

[13] I. H. Deutsch, J. C. Garrison, and E. M. Wright, J. Opt. Soc. Am. B 8, 1244 (1991).

[14] A. E. Siegman, Phys. Rev. A 39, 1264 (1989).

[15] C. Lamprecht and H. Ritsch, Phys. Rev. Lett. 82, 3787 (1999).

[16] C. Lamprecht, M. K. Olsen, M. Collett, and H. Ritsch, Phys. Rev. A 64, 033811 (2001).

[17] C. Lamprecht and H. Ritsch, Phys. Rev. A 65, 023803 (2002).

[18] S. A. Brown and B. J. Dalton, J. Mod. Opt. 49, 1009 (2002).

[19] P. Goldberg, P. W. Milonni, and B. Sundaram, Phys. Rev. A 44, 1969 (1991).

[20] S. M. Dutra and G. Nienhuis, Phys. Rev. A 62, 063805 (2000).

[21] A. M. Lindberg, G. P. Karman, K. Joosten, G. Nienhuis, and J. P. Woerdman, Opt. Commun. 153, 55 (1998).

[22] M. H. Rubin, Phys. Rev. A 54, 5349 (1996).

[23] Y. J. Lu and Z. Y. Ou, Phys. Rev. A 62, 033804 (2000).

[24] A. Aiello, M. P. van Exter, G. Nienhuis, and J. P. Woerdman, Opt. Commun. 213, 81 (2002).

[25] R. A. Campos, B. E. A. Saleh, and M. C. Teich, Phys. Rev. A 40, 1371 (1989).

[26] F. Singer, R. A. Campos, M. C. Teich, and B. E. A. Saleh, Quantum Opt. 2, 307 (1990). 
[27] B. Yurke, S. L. McCall, and J. R. Klauder, Phys. Rev. A 33, 4033 (1986).

[28] B. L. Schumaker, Phys. Rep. 135, 317 (1986).

[29] M. E. Peskin and D. V. Schroeder, Introduction to Quantum Field Theory, 2nd ed. (Perseus Books, Cambridge, 2001).

[30] L. Mandel and E. Wolf, Optical Coherence and Quantum Optics, 1st ed. (Cambridge University Press, Cambridge, England, 1995).

[31] C. W. Gardiner and C. M. Savage, Opt. Commun. 50, 173 (1984)

[32] R. J. C. Spreeuw, M. W. Beijersbergen, and J. P. Woerdman, Phys. Rev. A 45, 1213 (1992).

[33] E. Hecht, Optics, 4th ed. (Addison-Wesley, Reading, MA, 2002).

[34] M. Brunel, O. Emile, M. Alouini, A. L. Floch, and F. Bretenaker, Phys. Rev. A 59, 831 (1999).

[35] B. Yurke, Phys. Rev. A 29, 408 (1984).

[36] R. Loudon, The Quantum Theory of Light, 3rd ed. (Oxford University Press, New York, 2000).

[37] P. Grangier and J.-P. Poizat, Eur. Phys. J. D 7, 99 (1999).
[38] A. M. van der Lee, A. L. Mieremet, M. P. van Exter, N. J. van Druten, and J. P. Woerdman, Phys. Rev. A 61, 033812 (2000).

[39] M. Ueda and N. Imoto, Phys. Rev. A 50, 89 (1994).

[40] A. Aiello, D. Fargion, and E. Cianci, Phys. Rev. A 58, 2446 (1998).

[41] S. Reynaud, A. Heidmann, E. Giacobino, and C. Fabre, Progress in Optics, edited by E. Wolf (North-Holland, Amsterdam, 1992), Vol. XXX.

[42] D. Lenstra and S. H. M. Geurten, Opt. Commun. 75, 63 (1990).

[43] K. W. McVoy, L. Heller, and M. Bolsterli, Rev. Mod. Phys. 39, 245 (1967).

[44] E. J. Mason and N. C. Wong, Opt. Lett. 23, 1733 (1998).

[45] Z. Y. Ou, Phys. Rev. A 49, 4902 (1994).

[46] A. S. Marathay, J. Opt. Soc. Am. 55, 969 (1965).

[47] H. A. Haus and S. Kawakami, IEEE J. Quantum Electron. QE-21, 63 (1985).

[48] S. M. Barnett, C. R. Gilson, B. Huttner, and N. Imoto, Phys. Rev. Lett. 77, 1739 (1996). 